\title{
The IAP-antagonist ARTS initiates caspase activation upstream of cytochrome $C$ and SMAC/Diablo
}

\author{
N Edison ${ }^{1,2,4}$, D Zuri ${ }^{1,4}$, I Maniv ${ }^{1}$, B Bornstein ${ }^{1}$, T Lev ${ }^{1}$, Y Gottfried ${ }^{1}$, S Kemeny ${ }^{1}$, M Garcia-Fernandez ${ }^{3}$, J Kagan ${ }^{1}$ and S Larisch ${ }^{*, 1}$
}

ARTS (Sept4_i2) is a pro-apoptotic tumor suppressor protein that functions as an antagonist of X-linked IAP (XIAP) to promote apoptosis. It is generally thought that mitochondrial outer membrane permeabilization (MOMP) occurs before activation of caspases and is required for it. Here, we show that ARTS initiates caspase activation upstream of MOMP. In living cells, ARTS is localized to the mitochondrial outer membrane. In response to apoptotic signals, ARTS translocates rapidly to the cytosol in a caspase-independent manner, where it binds XIAP and promotes caspase activation. This translocation precedes the release of cytochrome $C$ and SMAC/Diablo, and ARTS function is required for the normal timing of MOMP. We also show that ARTS-induced caspase activation leads to cleavage of the pro-apoptotic Bcl-2 family protein Bid, known to promote MOMP. We propose that translocation of ARTS initiates a first wave of caspase activation that can promote MOMP. This leads to the subsequent release of additional mitochondrial factors, including cytochrome $C$ and SMAC/Diablo, which then amplifies the caspase cascade and causes apoptosis.

Cell Death and Differentiation (2012) 19, 356-368; doi:10.1038/cdd.2011.112; published online 26 August 2011

Apoptosis is important for regulating cell numbers and maintaining tissue homeostasis. The main executioners of apoptosis are caspases, a family of cysteine proteases that cleave substrates after aspartate. ${ }^{1}$ In the mitochondrial pathway, release of pro-apoptotic factors, including cytochrome C (cytoC) and Smac/Diablo (SMAC), from the mitochondrial intermembrane space (IMS) to the cytosol promotes caspase activation. This release requires mitochondrial outer membrane permeabilization (MOMP). ${ }^{2}$ A holoenzyme complex known as the 'apoptosome' is formed when cytoC is released from mitochondria and binds to apoptotic protease-related factor-1 (Apaf-1) to activate procaspase- $9 .^{3}$

The best-studied family of caspase inhibitors is the inhibitor of apoptosis (IAP) proteins. ${ }^{4}$ IAP proteins contain at least one baculoviral IAP repeat (BIR) domain which can directly interact with caspases and inhibit their apoptotic activity, a RING domain that bestows E3-ubiquitin ligase activity and an Ubiquitin-associated (UBA) domain, which enables the binding of polyubiquitin conjugates via lysine $63 . .^{5-7}$ X-linked IAP (XIAP) directly inhibits caspases-3, -7 and $-9 .^{8}$ XIAP is considered to be the most potent inhibitor of caspases in vitro, and elevated levels of this protein are found in human cancers. ${ }^{9}$ Although XIAP-null mice are viable, it was recently shown that loss of XIAP function causes elevated caspase-3 activity and sensitizes certain primary cells toward apoptosis. ${ }^{7}$ In dying cells, apoptosis can be overcome through the release of caspases from their binding to IAP proteins. ${ }^{10-13}$
Several mammalian XIAP antagonists have been identified, including SMAC, ${ }^{14,15} \mathrm{Omi} / \mathrm{HtrA} 2^{16}$ and ARTS. ${ }^{17,18} \mathrm{SMAC}$ and Omi/HtrA2 are located in the mitochondrial IMS, contain a conserved IAP-binding motif (IBM) and are released to the cytosol upon apoptotic induction. ${ }^{14,15}$ Genetic inactivation of SMAC and Omi/HtrA2 has failed to reveal any physiological requirement in apoptosis, IAP regulation or tumor suppression, ${ }^{19,20}$ possibly because of functional redundancy of different IAP antagonists in the mouse. ARTS (Sept4_i2), derived from the human Sept4 gene, does not contain an IBM, instead it uses unique sequences to bind XIAP. ${ }^{18}$ ARTS expression is frequently lost in acute lymphoblastic leukemia patients, indicating that it functions as a tumor suppressor protein. $^{21}$ Sept4/ARTS-null mice show accelerated tumor development, elevated XIAP levels and have stem cells with increased resistance to cell death, demonstrating a physiological role of ARTS for regulating XIAP levels and apoptosis in vivo. ${ }^{22,23}$ Furthermore, these phenotypes of Sept4/ARTSnull mice are suppressed by inactivation of XIAP, demonstrating that ARTS acts mainly by targeting XIAP, in vivo. ${ }^{22}$

It is generally thought that MOMP occurs before activation of caspases and is required for it. ${ }^{2,24}$ In this study, we provide evidence that ARTS initiates caspase activation upstream of MOMP. We show that ARTS is localized to the outer membrane of mitochondria (MOM). Following induction of apoptosis, ARTS rapidly translocates to the cytosol in a caspase-independent manner where it binds XIAP.

\footnotetext{
${ }_{1}^{1}$ Department of Biology, Faculty of Natural Sciences, Cell Death Research Laboratory, University of Haifa, Multi-Purpose Building, Mount Carmel, Haifa 31905, Israel; ${ }^{2}$ The B. Rappaport Faculty of Medicine, Technion - Israel Institute of Technology, Haifa 31096, Israel and ${ }^{3}$ Molecular Pathology Programme, Spanish National Cancer Research Centre (CNIO), Melanoma Laboratory, Madrid 28049, Spain

${ }^{*}$ Corresponding author: S Larisch, Department of Biology, Faculty of Natural Sciences, Cell Death Research Laboratory, University of Haifa, Multi-Purpose Building, Mount Carmel, Haifa 31905, Israel. Tel.: + 972544 314671; Fax: + 9724 8288763; E-mail: saritlarisch@gmail.com

${ }^{4}$ These authors contributed equally to this work.

Keywords: ARTS; apoptosis; caspases; XIAP; mitochondria

Abbreviations: BIR, baculoviral IAP repeat; CytoC, cytochrome C; IAP, inhibitor of apoptosis proteins; IF, immunofluorescence; IMS, intermembrane space; $\mathrm{KD}$, knockdown; MOM, mitochondrial outer membrane; MOMP, mitochondrial outer membrane permeabilization; PK, proteinase K; shRNA, short-hairpin RNA; STS, staurosporine; UPS, ubiquitin-proteasome system; XIAP, X-linked IAP

Received 18.2.11; revised 23.6.11; accepted 08.7.11; Edited by A Villunger; published online 26.8.11
} 
The translocation of ARTS from mitochondria precedes the release of cytoC and SMAC and leads to degradation of XIAP, but not cIAP1, before MOMP. Moreover, knockdown of ARTS inhibits the release of SMAC and cytoC, suggesting that ARTS is required for proper 'on time' release of these proteins. This early ARTS-induced caspase activation can promote the cleavage of the $\mathrm{BH} 3-$ containing protein Bid. We propose that ARTS translocation initiates a first wave of caspase activation through antagonizing XIAP, which leads to de-repression of caspases. According to our model, this initial caspase activity feeds back to mitochondria causing cleavage of Bid and possibly other factors to initiate MOMP, thereby amplifying the caspase cascade leading to apoptosis.

\section{Results}

ARTS is localized to the MOM. ARTS localizes to mitochondria of living cells and translocates to the cytosol in response to apoptotic stimuli. ${ }^{17,18}$ Other mitochondrial proapoptotic proteins, including cytoC and SMAC, reside in the IMS. $^{25,26}$ To gain more insight into the mechanism and kinetics by which ARTS is released from mitochondria, we determined the precise localization of ARTS within mitochondria using several distinct approaches. First, we used immunogold labeling with a monoclonal anti-ARTS antibody to visualize ARTS in COS-7 cells. Both endogenous and overexpressed ARTS were detected at the MOM (Figure 1a, white arrows). In order to investigate whether ARTS is readily accessible at the surface of intact mitochondria, mitochondrial fractions of COS-7 cells expressing ARTS were treated with increased concentrations of proteinase $\mathrm{K}(\mathrm{PK})$. We observed a reduction in ARTS levels at the highest $\mathrm{PK}$ concentration $(400 \mu \mathrm{g} / \mathrm{ml})$ similar to VDAC, a known MOM protein (Figure 1b). In contrast, Hexokinase I, a known peripherally associated protein, was completely digested with all concentrations of PK. Upon treatment with $1 \%$ Triton $X-100$, which solubilizes mitochondria membranes, all shown proteins were digested by even the lowest PK concentration $(50 \mu \mathrm{g} / \mathrm{ml})$ (Figure $1 \mathrm{~b})$. Therefore, by this criterion, ARTS appears to be localized to the MOM, similar to VDAC, and the association with MOM protects it from digestion by PK. Next, we treated mitochondrial fractions with alkaline carbonate buffer to determine whether ARTS behaves as a peripheral or integral MOM protein. ARTS, Bcl-XL and $\mathrm{Bcl}-2$ were resistant to carbonate extraction whereas Hexokinase I was carbonate sensitive (Figure 1c). Furthermore, SMAC and cytoC were released into the supernatant fraction during alkaline treatment (Figure 1c), consistent with their known localization in the IMS. ${ }^{25,26}$ Finally, treatment of the mitochondrial fraction with increasing concentrations of digitonin demonstrated again that ARTS behaves like an integral MOM protein, very similar to VDAC, Bcl-2 and Bcl-XL (Figure 1d).

Translocation of ARTS from mitochondria precedes the release of SMAC and cytoC. Overexpression of ARTS in certain cultured cells is sufficient to induce the release of cytoC from mitochondria and promote apoptosis. ${ }^{27}$ ARTS can also induce the release of SMAC to the cytosol (Figure 2a). Several reports have shown that the release of SMAC and cytoC occurs simultaneously. ${ }^{28}$ Therefore, we considered the possibility that the translocation of ARTS may precede the exit of SMAC and cytoC. In order to compare the kinetics by which endogenous ARTS and SMAC are released from mitochondria, we used HeLa cells that contain relatively high levels of endogenous ARTS and SMAC. Following treatment with staurosporine (STS), we detected cytosolic ARTS as short as 15-30 min after apoptosis induction (Figure 2c; Supplementary Figure S1c). The translocation of ARTS to the cytosol preceded that of SMAC and cytoC by several hours (Figure 2c; Supplementary Figures S1a, c and d). Similar results were seen in SH-SY5Y human neuroblastoma cells, and in mouse embryonic fibroblasts (Supplementary Figures S2a and b), and using UV irradiation in HeLa cells (Figure 6c). Importantly, early translocation of ARTS to the cytosol (30 min) was not blocked by the caspase inhibitor Q-VD, suggesting that it does not require caspase activity (Figure 2d). In contrast, at the later time point (180 min), presumably after MOMP, translocation of ARTS and SMAC was caspase dependent (Figure 2d). Next, to determine whether the early translocation of ARTS to the cytosol affects the release of SMAC and cytoC, we used HeLa cells in which ARTS expression was knocked down by short-hairpin RNAs (shRNAs) (ARTS KD HeLa) (Figure 2b; Supplementary Figure S1f). ARTS KD HeLa cells exhibited a clear reduction in the levels of three different apoptotic markers in response to STS; H2AX, cleaved PARP and active caspase-9 (Figure 2b). This suggests that lack of ARTS protects HeLa cells against apoptosis. Moreover, SMAC and cytoC were found in the cytosol of ARTS KD HeLa cells only after $6 \mathrm{~h}$ of STS induction (Figure 2c; Supplementary Figure S1a). Similar results were seen using immunofluorescence (IF) assay with mCherry-tagged ARTS and mCherry-tagged SMAC (Figures $3 a$ and $b$ ), demonstrating that loss of ARTS inhibits the release of SMAC to the cytosol. Next, we performed an IF assay with HeLa cells double stained for ARTS and SMAC (Figures 3c and $\mathrm{d}$ ). Background levels of apoptosis were detected for ARTS and SMAC at time 0 as a result of transfection. However, $>70 \%$ of cells showed cytosolic ARTS staining after $15 \mathrm{~min}$ and $>90 \%$ after 30 and $60 \mathrm{~min}$ of STS treatment. In contrast, a significant release of SMAC into the cytosol was only detected after $180 \mathrm{~min}$ of STS induction in $76 \%$ of cells, and a significant loss of mitochondrial SMAC staining was also observed at this time (Figure 3d). These results confirm our western blot data that translocation of ARTS to the cytosol precedes that of SMAC. Likewise, HeLa cells stably transfected with cytoC-GFP lost the typical mitochondrial staining for cytoC-GFP and endogenous SMAC after $3 \mathrm{~h}$ of STS treatment (Figure $4 \mathrm{a}$ ). In contrast, endogenous ARTS was detectable in the cytosol already at 15-30 min following STS induction (Figure 4b). To confirm that the early increase in levels of ARTS at the cytosol reflects the translocation of mitochondrial ARTS rather than newly synthesized ARTS which is not properly inserted into MOM, we performed a fractionation assay on HeLa cells in the presence or absence of inhibitor of protein 
a
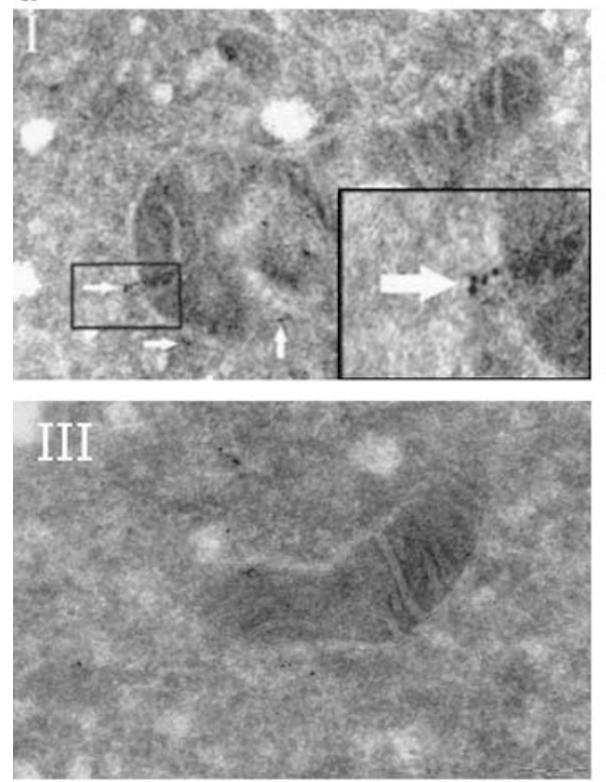

C

$\mathrm{Na} 2 \mathrm{CO} 3$

\begin{tabular}{|l|l|l|l|l|}
\hline M & P & S & P & S \\
\hline & + & + & - & - \\
\hline
\end{tabular}

HXKI

ARTS

Bcl-XL

Bcl2

Cyto $C$

SMAC

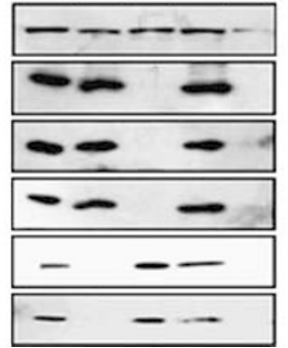

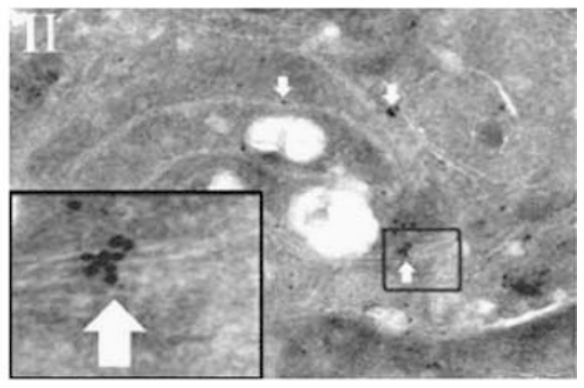

b

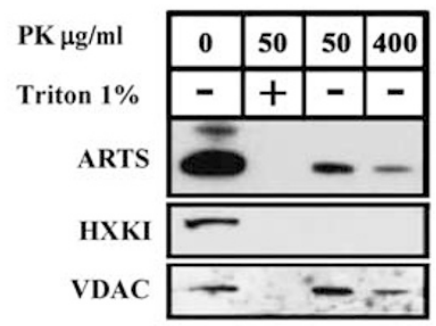

d

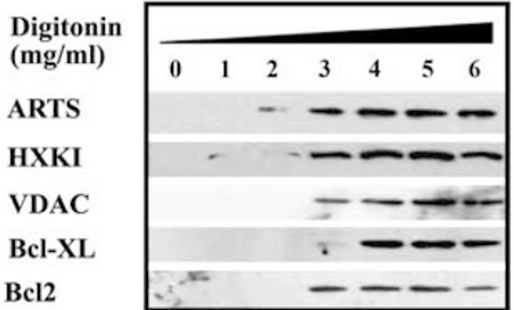

Figure 1 ARTS is localized to the MOM. (a) EM localization of ARTS. TEM images of ARTS immunogold labeling in COS-7 cells. Endogenous ARTS (I - left panel, Bar $-500 \mathrm{~nm}$ ) and exogenous ARTS (II - right panel, Bar $-200 \mathrm{~nm}$ ) are shown at the outer membrane of the mitochondria (white arrows). Staining using only secondary antibody was used as negative control (III, Bar $-200 \mathrm{~nm}$ ). (b) PK treatment of mitochondria shows that ARTS is located at the MOM. Purified mitochondria from COS-7 cells transfected with 6Myc-ARTS were treated with increased concentrations of PK and analyzed using SDS-PAGE and western blot analysis. ARTS levels were decreased upon treatment with elevated concentrations of PK, similar to VDAC, a well-known MOM integral protein. In contrast, Hexokinase I (HXKI) (an outer membrane-associated protein) was completely digested by PK. Upon treatment with 1\% Triton, which solubilizes mitochondrial membranes, all proteins examined were digested by the smallest PK concentration. (c) ARTS is tightly associated with the MOM. COS-7 cells were transiently transfected with 6Myc-ARTS. Fractions enriched for mitochondria were treated with sodium carbonate $(\mathrm{Na2} \mathrm{CO}) \mathrm{pH} 11.5$ or mock treated with sucrose. The whole untreated mitochondrial fraction $(\mathrm{M})$, supernatant $(\mathrm{S})$ and pellet $(\mathrm{P})$ fractions were analyzed by immunoblotting. In contrast to $\mathrm{HXKI}$, whose association with the MOM is interrupted by the carbonate treatment (found in the supernatant), ARTS was not detected in the supernatant fraction, similarly to $\mathrm{Bcl}-2$ and $\mathrm{Bcl}-\mathrm{XL}$. This suggests that ARTS behaves like an integral MOM protein, similar to Bcl-2 and Bcl-XL. CytoC and SMAC, which reside in the IMS, are released to the supernatant fraction during alkaline treatment. (d) ARTS behaves similar to MOM proteins in response to treatment with digitonin. Fractions enriched for mitochondria were obtained from COS-7 cells transfected with 6Myc-ARTS and incubated with the indicated concentrations of digitonin. Following centrifugation, the supernatant fraction was subjected to SDS-PAGE and western blot analyses. All shown proteins were not present in the supernatant fraction in the absence of digitonin. Translocation of ARTS from the mitochondrial pellet into the supernatant follows a similar pattern as seen for other integral MOM proteins, such as Bcl-2, Bcl-XL and VDAC

translation cycloheximide (CHX). Supplementary Figure S1ell demonstrates accumulation of ARTS in the cytosol of $\mathrm{CHX}$-treated cells indicating, that at least some, if not the majority of ARTS present in the cytosol following apoptotic induction, is caused by its rapid translocation from mitochondria. Together, these results reveal that ARTS translocates to the cytosol before cytoC and SMAC, and that the function of ARTS is required for the proper 'on time' release of cytoC and SMAC from mitochondria during apoptosis.
An ARTS-XIAP complex forms rapidly in response to apoptotic stimuli, and XIAP levels decrease before the release of cytoC and SMAC. ARTS can induce apoptosis, at least in part, by binding to XIAP, ${ }^{17}$ but the kinetics by which an ARTS-XIAP complex forms have not been determined. In order to visualize the interaction of ARTS and XIAP, we used the split-Venus, Bimolecular Fluorescence Complementation (BiFC) system. ${ }^{29}$ The fluorescent signal that serves as a measurement for ARTS-XIAP proximity was evaluated by IF (Figure 5a) and flow cytometric analyses (Figure 5b). Both 


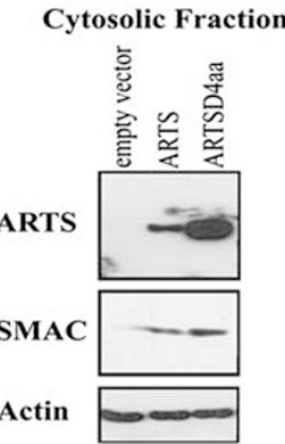

C

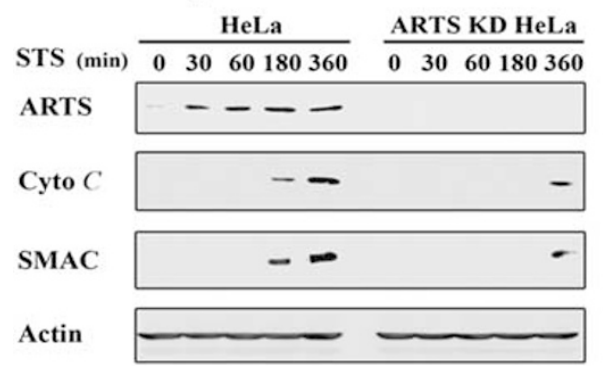

dII

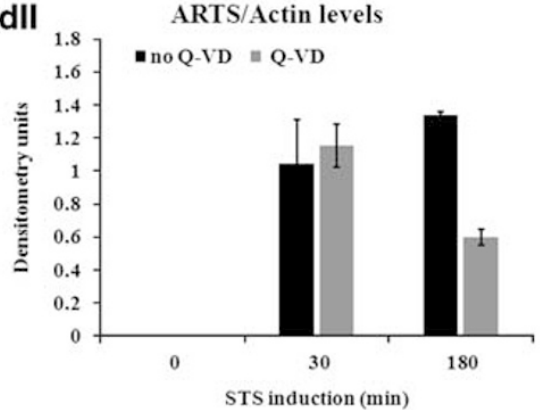

b

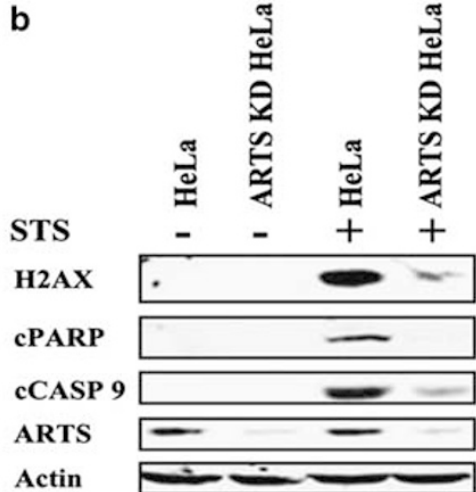

dl
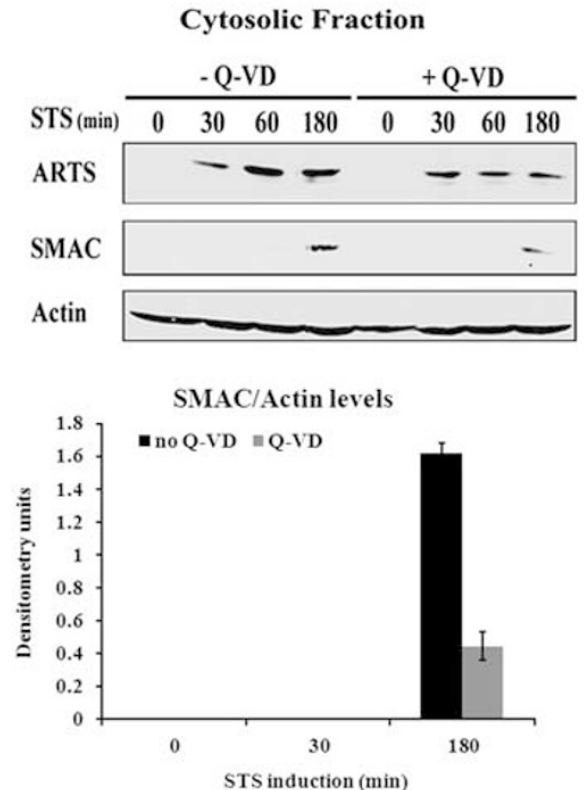

Figure 2 The translocation of ARTS from mitochondria precedes the release of cytoC and SMAC. (a) Overexpression of ARTS promotes the release of SMAC from mitochondria. Cellular fractionation assay performed on COS-7 cells transfected with AU5-ARTS and a mutant ARTS lacking its first 4aa (ARTSD4aa). Western blot analysis of the cytosolic fraction was conducted using the indicated antibodies. ARTS overexpression promotes the release of SMAC from mitochondria to the cytosol. (b) Knockdown of ARTS in HeLa cells caused resistance to STS-induced apoptosis. ARTS KD HeLa cells and control HeLa cells were incubated with STS $1.75 \mu \mathrm{M}$ for $3 \mathrm{~h}$. Cells were lysed and analyzed by western blot with the indicated antibodies. ARTS KD HeLa cells are protected against STS-induced apoptosis, exhibiting significant reduction in the levels of three different apoptotic markers: H2AX, cleaved PARP (cPARP) and CCASP 9. (c) The translocation of endogenous ARTS from mitochondria precedes the release of endogenous cytoC and SMAC in HeLa cells and requires for it. Cellular fractionation assays were performed on HeLa and ARTS KD HeLa cells. Cells were treated with $1.75 \mu \mathrm{M}$ STS for increasing periods of time and western blot analysis of the cytosolic fraction was conducted using the indicated antibodies. The translocation of endogenous ARTS from mitochondria to the cytosol was seen after $30 \mathrm{~min}$ following STS induction. This precedes the release of SMAC and cytoC, which was detected in the cytosol only after $3 \mathrm{~h}$ of STS induction. In ARTS KD HeLa cells, SMAC and cytoC were seen in the cytosol after $6 \mathrm{~h}$ of STS induction, suggesting that ARTS is required for proper release of cytoC and SMAC from the mitochondria. (d) The early translocation of endogenous ARTS from the mitochondria to the cytosol of HeLa cells occurs in a caspase-independent manner. (dI) Cell fractionation assays were performed on HeLa cells. Cells were treated with $1.75 \mu \mathrm{M}$ STS for increasing periods of time in the presence or absence of the pan-caspase inhibitor Q-VD. Western blot analysis of the cytosolic fraction was conducted with the indicated antibodies. The early translocation of endogenous ARTS from the mitochondria to the cytosol of HeLa cells (detected after $30 \mathrm{~min}$ ) preceded the release of SMAC and cytoC (detected after $180 \mathrm{~min}$ ) and was not inhibited by Q-VD. Treatment with Q-VD affected the cytosolic levels of ARTS and SMAC only after $3 \mathrm{~h}$ of STS treatment. (dII) Densitometry analyses of data shown in (dl) and repeating experiments demonstrating the levels of ARTS or SMAC in the cytosolic fraction of HeLa cells with or without the caspase inhibitor Q-VD. Measurements are presented relative to actin levels which served as loading controls

approaches show that fluorescence indicative of ARTS-XIAP complex formation can be seen as early as $15 \mathrm{~min}$ after STS treatment. The fluorescent signal continues to increase and peaks at $60 \mathrm{~min}$ after STS induction. Since we never observed the release of either cytoC or SMAC at time points earlier than $3 \mathrm{~h}$ after STS induction, we conclude that ARTS forms a complex with XIAP before MOMP.
We also investigated the levels of XIAP, clAP1 and other apoptotic proteins during STS treatment (Figure 6a), and in response to UV irradiation (Figure $6 \mathrm{c}$ ). This revealed that XIAP, but not clAP1 levels were decreased 30-120 min after STS induction. Over this time period, we also observed processing of caspase-9 (Figure 6a). Significantly, knockdown of ARTS blocked the decrease of XIAP levels and also 
a HeLa

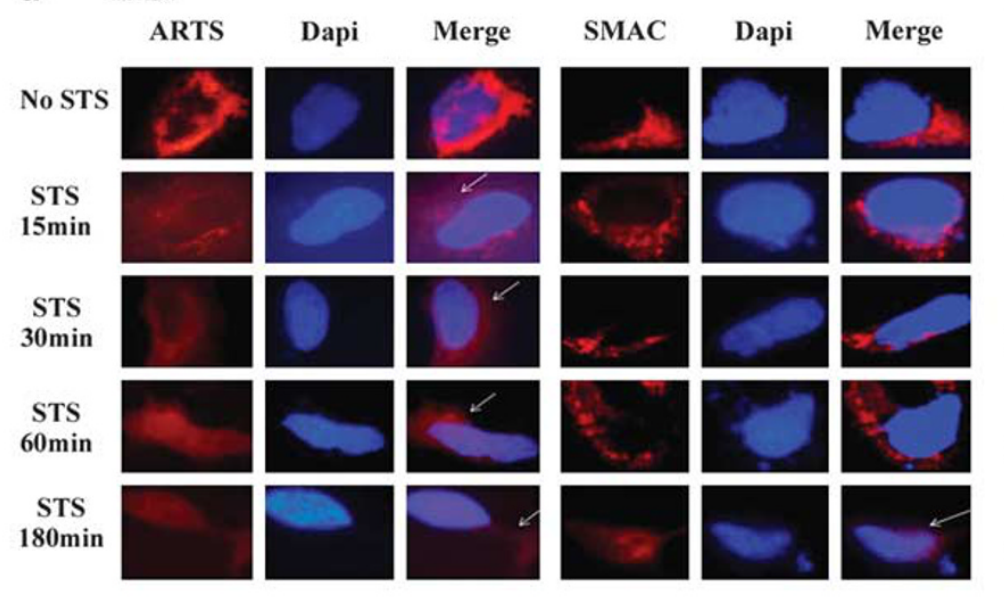

b

ARTS KD HeLa

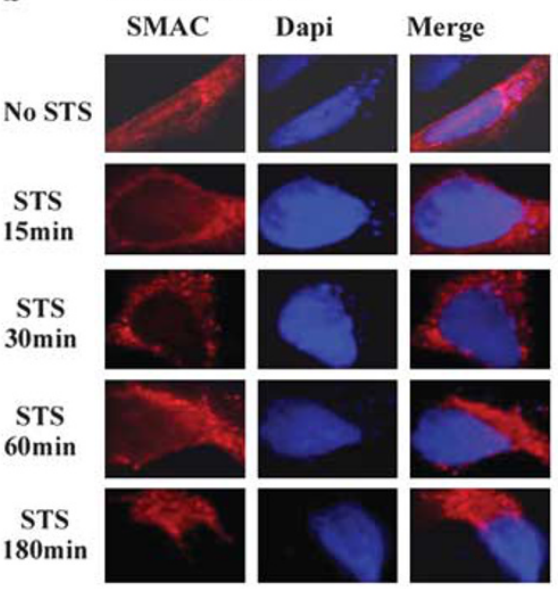

C
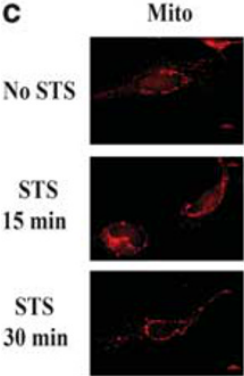

STS $60 \mathrm{~min}$

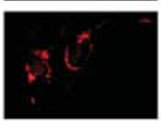

STS $180 \mathrm{~min}$

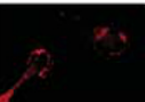

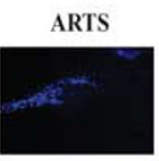
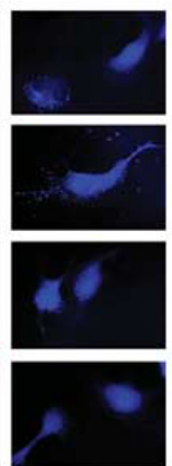
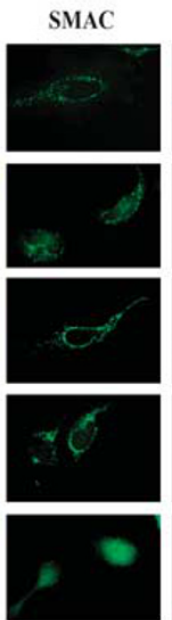
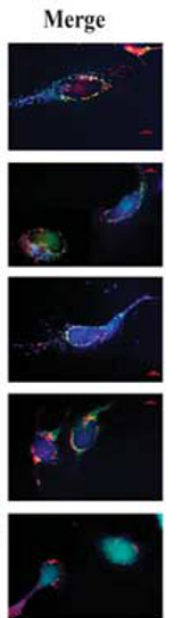

d

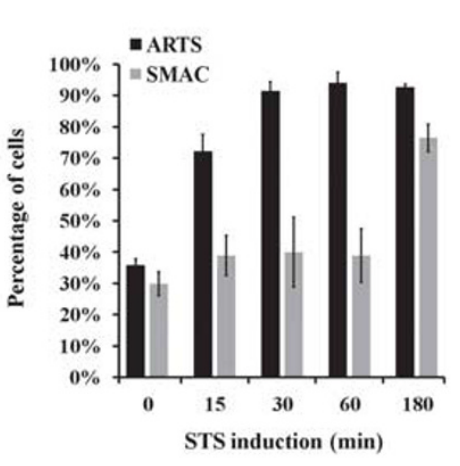

Mitochondrial localization

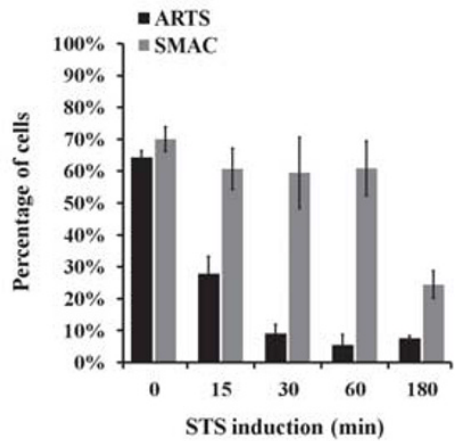

Figure 3 The translocation of ARTS from mitochondria precedes the release of SMAC. (a) The translocation of mCherry-ARTS from mitochondria precedes the release of mCherry-SMAC in HeLa cells. HeLa cells were transfected with mCherry-ARTS or mCherry-SMAC. Cells were treated with $1.75 \mu \mathrm{M}$ STS for the indicated time periods. Arrows indicate cytosolic staining pattern. Nuclei were stained blue with DAPI staining. ARTS loses its bright mitochondrial staining pattern and acquires a diffuse faint cytosolic staining as early as $15 \mathrm{~min}$ after STS treatment. In contrast, SMAC maintains its bright mitochondrial staining and exhibits a diffuse cytosolic pattern only after $3 \mathrm{~h}$ of STS induction. Thus, the translocation of mCherry-ARTS from the mitochondria to the cytosol (after $15 \mathrm{~min}$ ) precedes the release of mCherry-SMAC (seen only after $180 \mathrm{~min}$ ). (b) Downregulation of ARTS with shRNA in HeLa cells ('ARTS KD HeLa') inhibits the release of SMAC into the cytosol. ARTS KD HeLa cells were transfected with mCherrySMAC. Cells were treated with $1.75 \mu \mathrm{M}$ STS for the indicated time periods. Nuclear morphology was evaluated by DAPI staining. SMAC retained its bright mitochondrial pattern of staining in these ARTS KD cells, even after $3 \mathrm{~h}$ following STS treatment. These data suggest that ARTS is required for the efficient release of SMAC. (c) The translocation of ARTS from mitochondria to cytosol precedes the release of SMAC in transfected HeLa cells. HeLa cells were co-transfected with AU5-ARTS and flag-SMAC. Cells were also stained with mitotracker (red), and incubated with anti-ARTS (blue) and anti-SMAC (green) antibodies. The panel shows representative images from each time point (Bar $-10 \mu \mathrm{m})$. ARTS loses its colocalization with mitotracker and acquires a diffuse faint cytosolic staining as early as 15 min after STS treatment. In contrast, SMAC remains colocalized with mitotracker at time points 15,30 and $60 \mathrm{~min}$, and exhibits diffuse cytosolic pattern only after $3 \mathrm{~h}$ of STS induction. Thus, the translocation of ARTS from the mitochondria to the cytosol precedes the release of SMAC. (d) Quantification of the cellular localization of ARTS and SMAC. To quantify the number of cells with cytosolic versus mitochondrial localization of ARTS and SMAC, cells were transfected and IF double labeling was performed. The graphs represent the numbers of cells displaying cytoplasmic/mitochondrial staining as a percent of total counted cells (mean \pm S.E., $n=300$ ). Due to the transfection, there were always cells undergoing apoptosis, causing background for both ARTS and SMAC already at time 0. However, a significant increase in the proportion of cells with cytoplasmic staining and corresponding loss of mitochondrial localization of ARTS was repeatedly seen as soon as 15 min after STS induction. These results confirm our western blot data that translocation of ARTS to the cytosol precedes that of SMAC

delayed caspase processing (Figures $6 a$ and $b$ ). Although the signal representing the ARTS-XIAP complex increases for the first hour after induction of apoptosis (Figures $5 a$ and b), XIAP protein levels decline (Figure 6a). This indicates that the fraction of XIAP that is bound to ARTS increases during the first hour of apoptosis induction while there is concomitant degradation of total XIAP protein. By $180 \mathrm{~min}$ after STS induction, the ARTS-XIAP-Venus signal is significantly decreased, presumably due to the overall reduced XIAP levels. This suggests that a complex between ARTS and XIAP forms rapidly upon induction of apoptosis and before MOMP, and that the early function of ARTS promotes caspase activation.

The early translocation of ARTS to the cytosol promotes activation of caspase-9 and cleavage of Bid. Cleavage of the pro-apoptotic Bcl-2 family member Bid by caspases has been shown to promote MOMP and the release of cytoC. ${ }^{30,31}$ 
a Endogenous
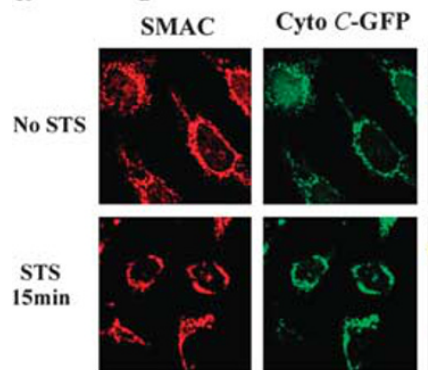

Merge
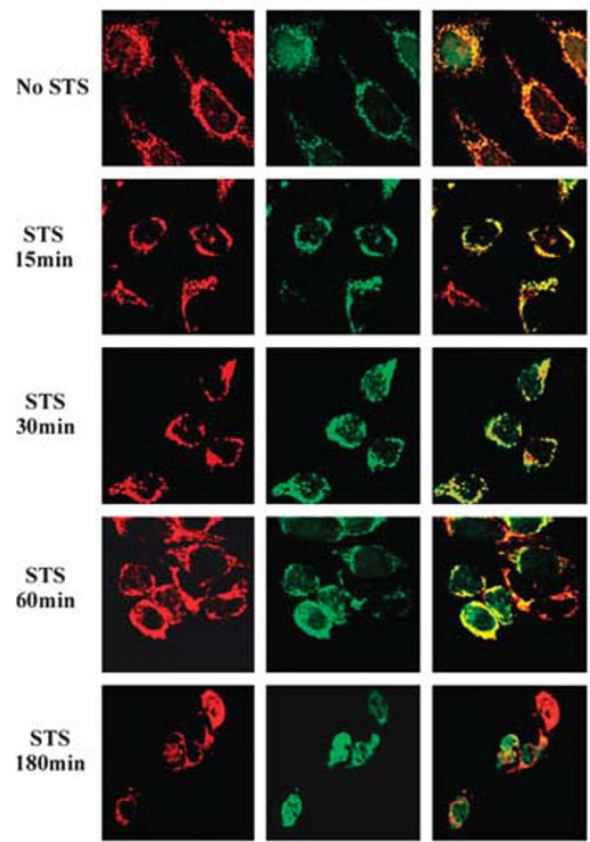

b Endogenous

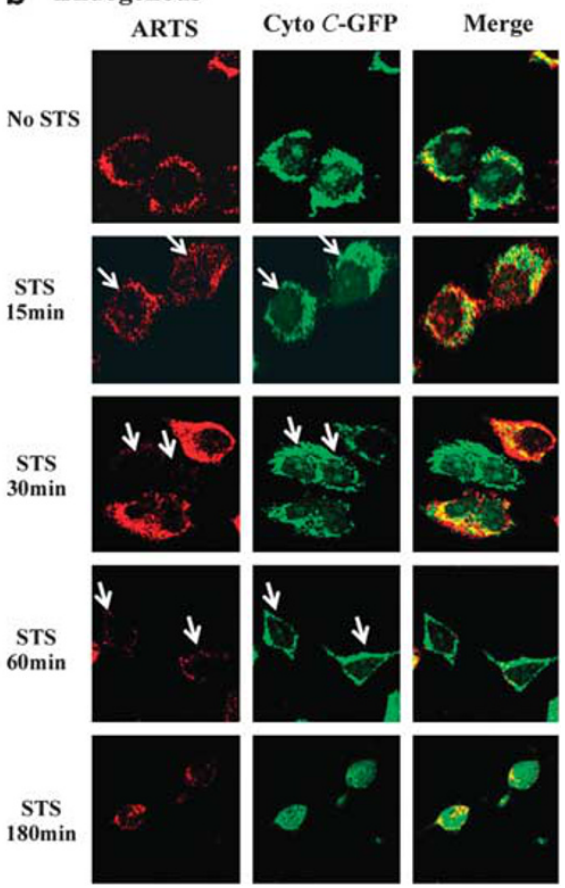

Figure 4 The translocation of endogenous ARTS from mitochondria precedes the release of endogenous SMAC in HeLa cells stably transfected with cyto C-GFP. (a and b) IF assays were performed on HeLa cells stably transfected with cytoC-GFP. Endogenous SMAC (a, red label) exhibited a loss of its condensed mitochondrial colocalization with cytoC-GFP (green) only after $3 \mathrm{~h}$ of STS treatment. In contrast, endogenous ARTS (b, red label) showed a loss of its mitochondrial colocalization with cytoC-GFP after as early as 15 min of STS treatment. White arrows in (b) mark cells in which cytoC-GFP is still displaying mitochondrial, condensed staining while ARTS has already translocated to the cytosol. Although some cells showed a release of cytoC-GFP after $1 \mathrm{~h}$, most of the cells showed cytosolic diffused staining only after $3 \mathrm{~h}$

To determine whether ARTS influences MOMP, and the release of cytoC and SMAC via Bid, we examined the levels of truncated Bid (tBid) in HeLa and ARTS KD HeLa cells following STS treatment. Bid levels are reduced $30 \mathrm{~min}$ after UV induction (Figure 6c) and tBid appears $60 \mathrm{~min}$ after STS induction (Figure 6a). Since the reduction of Bid levels is blocked by caspase inhibitors we attribute it to caspase cleavage (Figure 6d). Furthermore, only small amounts of tBid were seen 180 min after STS induction in ARTS KD HeLa cells, indicating that ARTS is required for promoting the observed activation of Bid (Figure 6a). Importantly, the presence of cleaved caspase- 9 was demonstrated in HeLa cells, 30 and $60 \mathrm{~min}$ after STS and UV treatment (Figures $6 a-c$, respectively) and caspase- 9 activity was demonstrated 60 min following STS induction (Figure 6b). Interestingly, in ARTS KD HeLa cells activation of caspase-9 was significantly delayed (Figure 6a), and activation of caspase3 could not be detected (Figures $6 a$ and $c$ ). Caspase- 2 and caspase-3 activation was detected only after $3 \mathrm{~h}$ in HeLa cells (Figures $6 \mathrm{a}$ and $\mathrm{c}$ ). We could not detect activation of caspase-8 following STS treatment (data not shown). Caspase-9 was shown to promote truncation of Bid. ${ }^{32}$ Thus, it seems that ARTS can promote MOMP, at least in part, through inducing caspase- 9 activation and cleavage of Bid. An early decrease in XIAP and Bid, but not in clAP1 levels was seen $30 \mathrm{~min}$ after STS as well as UV induction (Figures $6 \mathrm{a}$ and $\mathrm{c}$ ). This early reduction of XIAP is associated with caspase-9 activation and Bid cleavage (Figures 6a-c) and ARTS seems to be required for it (Figures $6 a$ and $b$ ).
Downregulation of clAP1 was seen only after 180 and $360 \mathrm{~min}$ of STS treatment (Figures $6 \mathrm{a}$ and $8 \mathrm{c} 1$ ). We, therefore, suggest that de-repression of caspase- 9 occurring $30-60 \mathrm{~min}$ following apoptotic induction by binding of ARTS to XIAP, can lead to cleavage of Bid which is known to feedback to mitochondria and induce MOMP and the release of cytoC.

Inactivation of ARTS in HeLa cells leads to resistance toward apoptosis. To assess cell death in HeLa versus ARTS KD HeLa cells, we have performed several assays including XTT assay, counting of DAPI-stained nuclei, clonogenic survival assay and counting of terminal deoxy nucleotidyltransferase-mediated deoxyuridine triphosphate nick end-labeling (TUNEL)-positive cells (Figure 7). All four different assays showed reduced cell death in ARTS KD cells following treatment with STS when compared with wt HeLa (Figures $7 a-d$ ). These data support our hypothesis that early activation of caspases induced by ARTS promotes cell death.

ARTS promotes rapid and specific degradation of XIAP but not clAP1 protein upon induction of apoptosis. Upon induction of apoptosis, XIAP protein is degraded by the ubiquitin-proteasome system (UPS). ${ }^{4}$ SMAC and/or smallmolecule derivatives ('SMAC-mimetics') selectively reduce the levels of cIAP1 and clAP2, but not that of XIAP. ${ }^{33}$ ARTS can bind to multiple IAP family members; clAP1 (Figure 8a), ML-IAP (Livin) (data not shown) and XIAP (Figures 5a, b and 

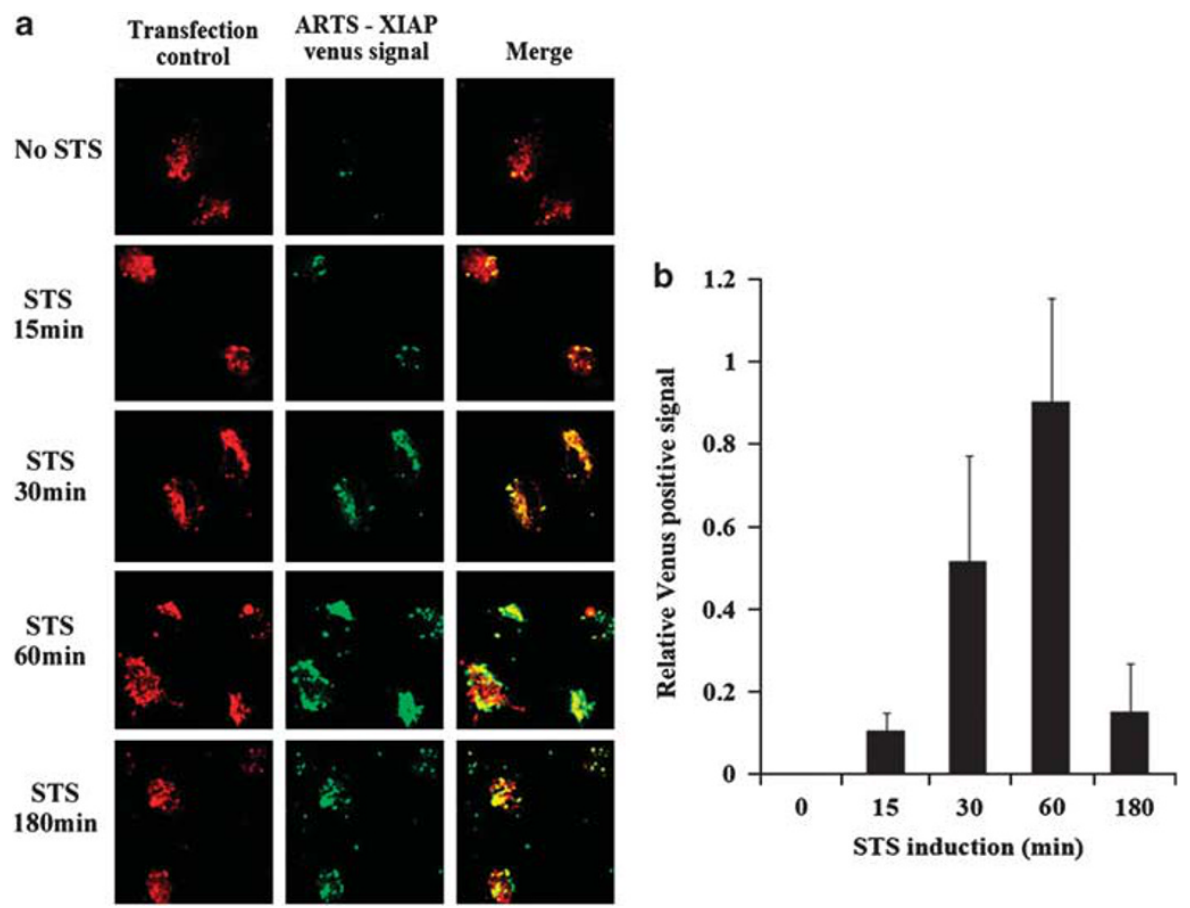

Figure 5 An ARTS-XIAP complex forms rapidly in response to apoptotic stimuli (BiFC assay). HeLa cells were transiently transfected with ARTS and XIAP fused to parts of Venus fluorescent protein (VN 1-173 and VC 1-155, respectively); pdsRED plasmid was used as a transfection efficiency marker. Apoptosis was induced with STS treatment and the fluorescent signal indicating the ARTS-XIAP proximity was measured by IF (a) and flow cytometric analyses of single-cell suspensions (b). (a) Cells assayed by IF were visualized by confocal microscopy (Zeiss LSM 510). (b) FACS results were normalized to the readings of transfection efficiency reporter (pdsRED) relative to $t=0$. The values represent mean values + S.E. of three independent experiments. The $Y$ axis represents the ratio between YFP fluorescence (reflecting binding of ARTS to XIAP) and the red fluorescence (marking the transfected cells) relatively to $t=0$. This ratio was calculated for each time point. Both IF and FACS analysis agree that fluorescence indicative of ARTS-XIAP complex formation can be observed as early as $15 \mathrm{~min}$ after STS treatment and that the fluorescent signal continues to increase and peaks at 60 min after STS induction

8a; Gottfried et $a{ }^{17}{ }^{17}$ ). Therefore, we investigated the possibility that ARTS promotes degradation of IAPs. First, we overexpressed ARTS and found this to strongly reduce endogenous XIAP protein levels, but not clAP1 (Figure 8b). Next, we treated HeLa cells with STS and evaluated XIAP and clAP1 levels at different incubation times (Figure 8cl). We saw that XIAP steady-state protein levels began to decrease already $30 \mathrm{~min}$ after STS induction, and this decrease continued for several hours. In contrast, there was no detectable change in clAP1 protein levels over the 3 -h induction (Figure $8 \mathrm{cl}$ ). Moreover, incubation of the cells with the proteasome inhibitor MG132 blocked most of the decline of XIAP protein. This indicates that XIAP is degraded by the UPS in cells stimulated to undergo apoptosis, consistent with earlier reports (Figure 8cl; Salvesen and Duckett ${ }^{4}$ ). In order to investigate the function of endogenous ARTS for controlling XIAP levels, we compared XIAP and cIAP1 levels in HeLa versus ARTS KD HeLa cells treated with STS (Figure 8cll). We found that knockdown of ARTS blocked the decrease of XIAP protein almost as well as MG132 (Figure 8cll). Taken together, our results suggest that ARTS is required for the rapid, early reduction of XIAP in response to STS treatment.

\section{Discussion}

The release of pro-apoptotic mitochondrial factors, such as cytoC and SMAC, has been traditionally viewed as the initiation stage of the mitochondrial pathway, promoting caspase activation. This redistribution of cytoC and SMAC from mitochondria to the cytosol requires MOMP. ${ }^{24}$ Although several studies indicate that the release of SMAC and cytoC can occur independently of caspases, ${ }^{34}$ others suggest that caspase activity is required for this. ${ }^{35,36}$ Here, we provide evidence that the mitochondrial IAP-antagonist ARTS may be the 'missing link' enabling MOMP, and the translocation of cytoC and SMAC in paradigms where this release depends on caspase activation. In particular, we suggest that ARTS has a critical role in initiating the mitochondrial apoptotic pathway upstream of MOMP, and that it acts by a different mechanism than other known IAP antagonists.

The first phase of ARTS translocation from mitochondria as well as binding of ARTS to XIAP occurs in a caspaseindependent manner (Figure 2d; Gottfried et al. ${ }^{17}$ ). Unlike SMAC, which resides in the IMS, ${ }^{25}$ ARTS is localized to the MOM (Figures 1a-d). Since ARTS does not contain any known trans-membrane domain, ARTS is either partially or fully embedded in the MOM, or it is covalently linked to integral MOM proteins. The localization of ARTS is highly relevant for understanding the kinetics by which ARTS translocates from mitochondria compared with SMAC and cytoC.

Despite a large number of studies, the precise timing of the release of SMAC and cytoC from mitochondria remains controversial. Several reports concluded that SMAC exits the mitochondria simultaneously with cytoC, ${ }^{28}$ while others have argued that the release of cytoC precedes that of SMAC. ${ }^{37}$ 
a

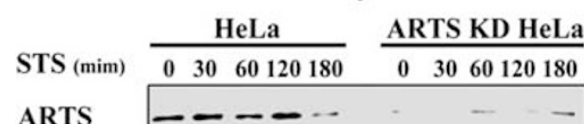
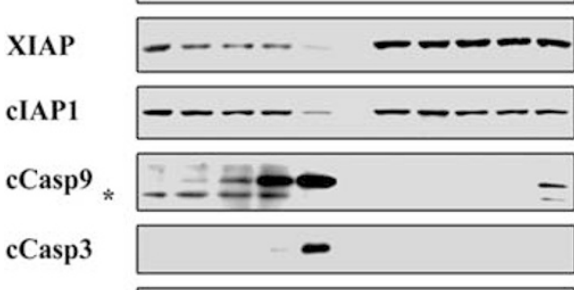

cCasp2

tBid

GAPDH

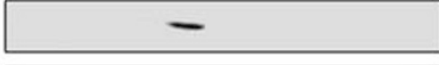

c

Time (min)

ARTS

XIAP

cIAP1

Bid

cCasp9

cCasp3

SMAC

Cyto $C$

Actin b

Caspase-9 Activity Assay

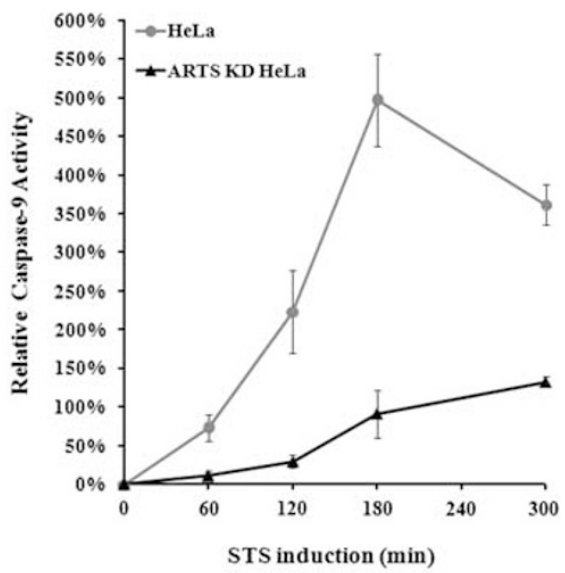

d

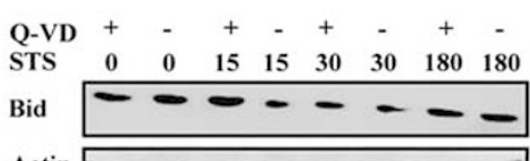

Actin

Figure 6 ARTS is required for early downregulation of XIAP, proper activation of caspases-9, 3 and 2 and truncation Bid. (a) Reduction in XIAP levels, activation of caspase-9 (cCASP9) and truncation of Bid are seen in HeLa cells 30-60 min after STS treatment, but not in ARTS KD HeLa cells. Thirty minutes after STS induction, XIAP but not cIAP1 levels were decreased. After $3 \mathrm{~h}, \mathrm{XIAP}$ and cIAP1 levels were severely reduced. Processing of caspase- 9 is seen already after 30 min of STS induction. tBid is seen 60 min after STS induction. Importantly, knockdown of ARTS blocked the decrease of XIAP as well as truncation of Bid and significantly inhibited the activation of caspase-9, 3 and 2. ${ }^{*}$ Indicates a non-specific band. (b) Caspase-9 activation is significantly compromised in ARTS KD HeLa cells. The cleavage of fluorescently labeled substrate of caspase-9 (Caspase-9 Fluorometric Assay Kit, BioVision, K118), caspase-9 activity assay revealed activation of caspase-9 as early as 60 min after STS treatment in HeLa cells with a peak of activity (5-fold increase) shown $180 \mathrm{~min}$ after apoptotic induction. Significant inhibition of caspase-9 activity is seen in ARTS KD HeLa cells. (c) Translocation of ARTS precedes the release of CytoC and SMAC following UV-induced apoptosis. Cells were irradiated with UV and the levels of various apoptotic proteins were measured after increasing periods of time. The levels of XIAP, cIAP1, Bid, cleaved caspase-9 (cCASP 9), cleaved caspase-3 (cCASP 3), SMAC and cytoC were analyzed using western blot analysis. An early decrease in XIAP, but not in cIAP1 levels was seen 30 min after UV induction. A second phase of protein downregulation was observed $6 \mathrm{~h}$ after UV irradiation. At this time period, cIAP1 levels as well as those of XIAP and Bid were severely reduced. (d) Bid levels are reduced as early as 15-30 min after STS treatment. HeLa cells were treated with $1.75 \mu \mathrm{M}$ STS for increasing periods of time in the presence or absence of the pan-caspase inhibitor Q-VD. Western blot analysis revealed that the early reduction in Bid levels occurring 15-30 min after STS treatment is blocked by caspase inhibitors. Thus, the observed reduction in Bid levels seems to occur as a result of caspase cleavage

We now show that ARTS translocation precedes the release of both cytoC and SMAC (Figures 2c, 3a, c and 4b; Supplementary Figures S1a, c and d), and that downregulation of ARTS significantly delays the release of both proteins (Figures 2c and 3b; Supplementary Figure S1a). Therefore, ARTS is required for the normal 'on time' release of cytoC and SMAC and functions upstream of MOMP.

Consistent with the idea of a biphasic action of ARTS in antagonizing XIAP, we found a progressive release of ARTS from the MOM (Supplementary Figure S1b). Both XIAP and
clAP1 levels are downregulated in cells undergoing apoptosis. ${ }^{4}$ We confirmed these results and also found a coordinated decrease in both XIAP and cIAP1 levels 3-6 h after apoptosis induction (Figures $6 \mathrm{a}, \mathrm{c}$ and $8 \mathrm{c}$ ). However, we observed a selective reduction of XIAP, but not cIAP1, at a much earlier stage, 30 min of STS and UV induction (Figures 6a, c and 8c). This early reduction of XIAP protein was blocked by knockdown of ARTS in HeLa cells (Figures $6 \mathrm{a}$ and $8 \mathrm{cll}$ ) and in Sept4/ARTS-null mice, ${ }^{22}$ suggesting that the early reduction of XIAP can be mainly attributed to ARTS. We also observed a 

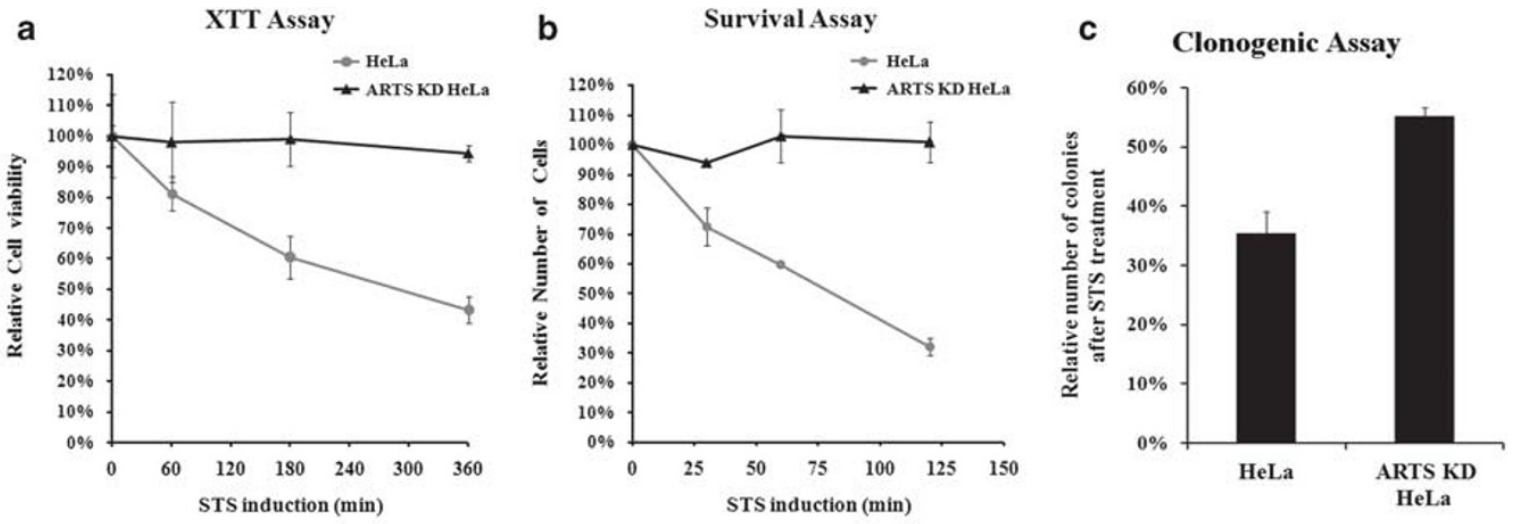

dl

HeLa
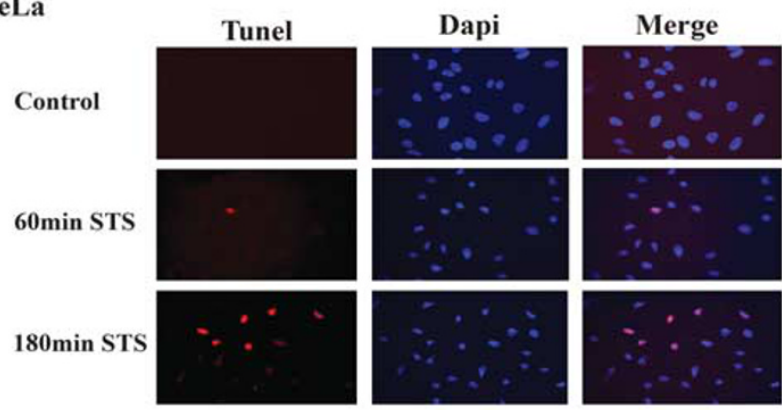

ARTS KD HeLa
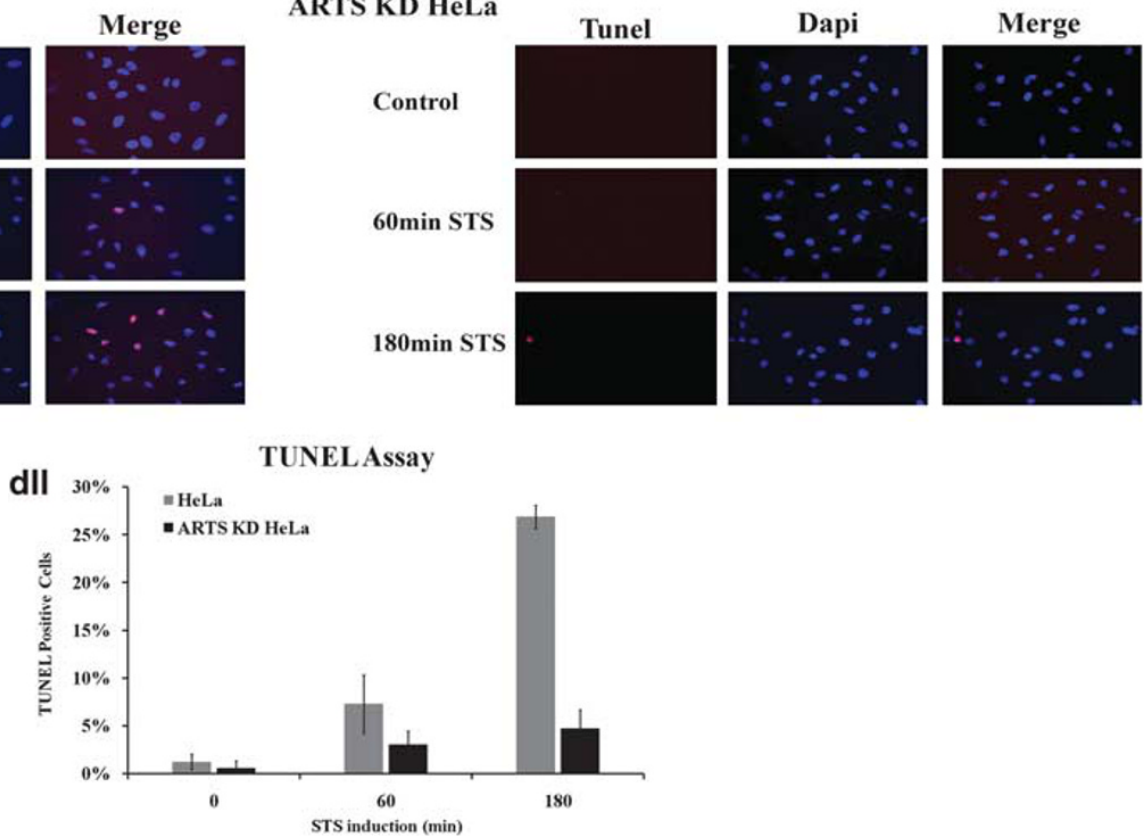

Figure 7 Inactivation of ARTS in HeLa cells leads to resistance toward apoptosis. (a) Knockdown of ARTS in HeLa cells results in increased viability of cells following STS induction-XTT results. Cell viability was quantified using XTT-based assay (for details, see Materials and Methods). ARTS KD HeLa cells showed a significant increase in cell survival compared with HeLa controls in response to STS (mean \pm S.E., $n=4$ ). (b) Knockdown of ARTS in HeLa cells results in increased viability of cells following STS induction counting of DAPI-stained number of cells. Quantification analysis of the cell survival after STS induction in HeLa versus ARTS KD HeLa cells was performed. Survival rates were determined by number of cells stained with DAPI. Cell survival numbers are composed of average cell counts of eight different fields for each sample using fluorescence microscope. The results showed higher numbers of ARTS knockdown cells at the various time points following STS treatment. (c) Knockdown of ARTS increases clonogenic survival. Apoptosis was induced in HeLa and ARTS KD HeLa cells by $1.75 \mu \mathrm{M} \mathrm{STS}$ for $1 \mathrm{~h}$. Cells were washed and 300 cells of each group were seeded into $6 \mathrm{~mm}$ dishes and allowed to grow for 12 days. Colonies $>50$ cells were counted. The graph shows the relative number of colonies after apoptotic induction. (d) Knockdown of ARTS in HeLa cells results in increased viability of cells following STS induction-TUNEL assay. Apoptosis rate was measured by percent of TUNEL-positive cells out of total number of cells after STS induction relatively to time 0 (mean \pm S.E., $n=3$ ). Altogether, results obtained using four different cell death assessing methods suggest that inactivation of ARTS in HeLa cells leads to resistance toward apoptosis. These data support our hypothesis that early activation of caspases induced by ARTS promotes cell death

consistent increase in XIAP levels in different tissues from Sept4/ARTS-null mice (data not shown). In contrast, clAP1 levels were not altered in these mice. This demonstrates that ARTS has a physiological and selective role for determining XIAP protein levels in vivo. Treatment of cells with the proteasome inhibitor MG132 blocked ARTS-induced reduction of XIAP levels, indicating that ARTS promotes degradation of XIAP via the UPS (Figure $8 c$ ). XIAP is an ubiquitin-ligase and its E3-ligase activity is important for both self-conjugation and caspase regulation. ${ }^{4,7}$ Therefore, ARTS may stimulate auto-ubiquitination of XIAP, or promote degradation of XIAP by activating another E3 ligase involved in this process, such as Siah1. ${ }^{38}$ We also observed cleavage of XIAP hours before MOMP in HeLa, but not in ARTS KD HeLa, suggesting that ARTS is required for caspasedependent cleavage of XIAP (data not shown).

Pro-apoptotic Bcl-2 family members, such as Bax, Bak and Bid, promote MOMP and the release of pro-apoptotic mitochondrial proteins. ${ }^{39}$ Bid can be cleaved by caspases-3, -9 and -8 , and cleaved Bid promotes MOMP. ${ }^{31,32}$ Therefore, 
a

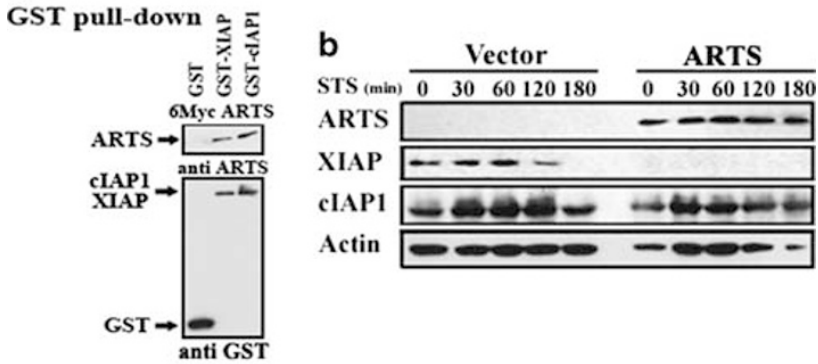

cl HeLa

MG132 - + - + - + - +

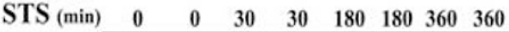

XIAP $-\cdots-\cdots--$

cIAP1

Actin

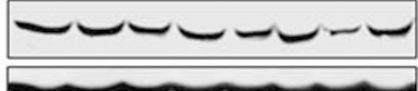

cll ARTS KD HeLa

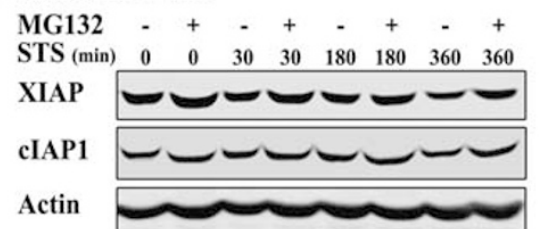

Figure 8 ARTS promotes rapid and specific degradation of XIAP but not of cIAP1 protein upon induction of apoptosis. (a) ARTS binds to both XIAP and CIAP1. COS-7 cells were transiently transfected with pSC2-6myc-ARTS construct together with mammalian GST-XIAP, GST-cIAP1 or GST vector (pEBG) alone as a control. The cells were treated with STS for $1 \mathrm{~h}$ and GST pull-down was carried out, followed by western blot analysis with anti-GST and anti-ARTS antibodies. ARTS binds to both cIAP1 and XIAP. (b) High levels of ARTS alone cause downregulation of XIAP levels, but not of cIAP1. COS-7 cells were transiently transfected with $\mathrm{pSC2}$ 6 myc-ARTS construct. The cells were treated with $1.75 \mu \mathrm{M}$ STS for increasing periods of time. Western blot analysis was carried out using the indicated antibodies. High levels of ARTS alone cause downregulation of XIAP levels, but not of CIAP1. (c) ARTS induces degradation of XIAP by the UPS. (cl) XIAP and CIAP1 endogenous protein levels were evaluated by western blot analysis of lysates from HeLa cells following different incubation times with STS. XIAP protein steadystate levels began to decrease $30 \mathrm{~min}$ after STS induction, and this decrease continued for several hours. In contrast, no detectable change in cIAP1 protein steady-state levels was seen over the next 3 -h time course. Moreover, incubation of the cells with the proteasome inhibitor MG132 $(20 \mu \mathrm{M}$ for $6 \mathrm{~h})$ blocked most of the decline of XIAP protein. Thus, XIAP is degraded by the UPS in cells stimulated to undergo apoptosis. (cll) ARTS is required for the rapid, early reduction of XIAP in response to STS treatment. XIAP and CIAP1 protein levels were determined using western blot analysis in ARTS KD HeLa cells treated with STS. Knockdown of ARTS blocked the decrease of XIAP protein almost as well as MG132. This suggests that ARTS is required for the rapid, early reduction of XIAP in response to STS treatment

tBid is one attractive candidate by which ARTS-induced caspase activation may feedback to mitochondria and promote MOMP. Consistent with a possible role of Bid, caspase-mediated cleavage of Bid was observed $60 \mathrm{~min}$ following STS induction (Figure 6a). This effect was significantly delayed in ARTS KD HeLa cells, suggesting that ARTS is required for the early cleavage of Bid. However, it is unlikely that Bid is the only target for ARTS-induced MOMP.
Model for the role of ARTS in initiating caspase activation and apoptosis

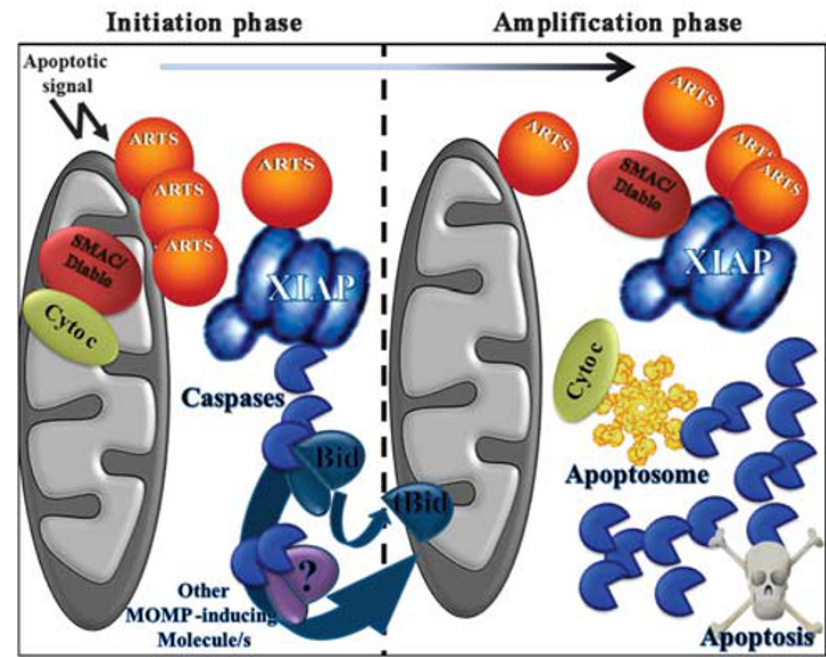

Figure 9 Model for the role of ARTS in initiating caspase activation and apoptosis. In living cells, low levels of ARTS-XIAP complexes form and contribute to XIAP turnover. Upon pro-apoptotic signals, ARTS translocates from the MOM to the cytosol and complex formation with XIAP dramatically increases. This causes a reduction of XIAP levels due to a combined of UPS-mediated degradation and caspase cleavage. This early reduction of XIAP triggers an initial, non-lethal wave of caspase activation (Phase I - Initiation phase). This initial caspase activity leads to the cleavage of Bid and presumably other factors that promote MOMP. Upon MOMP additional pro-apoptotic factors, including cytoC and SMAC are released together with more mitochondrial ARTS. Our model predicts that this subsequent release of ARTS, together with cytoC and SMAC, triggers a second and amplified wave of caspase activation which brings about the final destruction of the cell (Phase II - Amplification phase)

Several recent publications suggest that initiation of apoptosis in mammalian cells can occur through de-repression of caspases from their binding to IAP proteins. ${ }^{12,13}$ Our results provide a novel perspective to explain this phenomenon. The location of ARTS at the MOM may explain why ARTS translocates to the cytosol in a caspase-independent manner, before other mitochondrial pro-apoptotic proteins, which reside in the IMS.

In summary, we show that ARTS inhibits XIAP by a mechanism that is distinct from any other known mammalian IAP antagonist, ${ }^{40}$ and that it has an important role in initiating the mitochondrial pathway. We suggest that an initial caspase-independent exit of ARTS from mitochondria acts rapidly to specifically reduce XIAP protein levels. According to our model (Figure 9), this causes direct activation of low, nonlethal levels of caspase activity (Figure 6) and reduces the threshold toward apoptosis. Upon sustained apoptotic stimuli, elevated caspase levels are expected to feedback to mitochondria, at least in part by cleaving Bid, and promote MOMP, thereby initiating the non-reversible amplified phase of apoptosis (Figure 9). The exact mechanism by which caspase activation promotes MOMP remains to be elucidated, but this is beyond the scope of this study. Because ARTS acts very early in the initiation of a caspase cascade and is lost in human cancers, ARTS-based drugs may offer new opportunities to manipulate apoptosis for therapeutic purposes. 


\section{Materials and Methods}

Antibodies. Antibodies to the various proteins were purchased from the indicated companies, and used as instructed. Importantly, unless noted otherwise, in all our assays we used the monoclonal anti-ARTS antibody (Sigma-Aldrich, Saint Louis, MO, USA), which is the only currently commercially available antibody directed against the unique $\mathrm{C}$-terminus of ARTS. Antibodies against Hexokinase I (G-1, sc-46695), VDAC (N-18, sc-8828), Bcl-XL (A-20, sc-7122), Bcl-2 (N-19, sc-492), GAPDH (FL-335, sc-25778) and GST (B-14, sc-138) were purchased from Santa Cruz (Santa Cruz, CA, USA); antibodies against SMAC (\#567365) and H2AX (DR1016) are from Calbiochem (La Jolla, CA, USA). Additionally, antibodies against cytoC (2050_9, Clontech, Mountain View, CA, USA), XIAP (\#610716, BD, San Jose, CA, USA), clAP (AF818, R\&D Systems, Minneapolis, MN, USA), Bid (ab2561, Abcam, Cambridge, UK; \#2002, Cell Signaling), cleaved Caspase-9 (D315, \#9505, Cell Signaling, Beverly, MA, USA), cleaved Caspase-3 (D175, \#9661, Cell Signaling), Caspase-2 (ADI-905-685, Enzo Life Sciences, Plymouth Meeting, PA, USA), PARP (2660, SA-253, Biomol, Tebu, Le Perray en Yvelines, France) and actin (\#69100, MP Biomedicals, Solon, OH, USA) were used.

Mammalian cell cultures and various treatments. COS-7, HeLa cells were grown in Dulbecco's modified Eagle medium (DMEM) with $4.5 \mathrm{~g} / \mathrm{l} \mathrm{D}$-glucose. All media were supplemented with $10 \%$ fetal calf serum (FCS), penicillin $100 \mathrm{U} / \mathrm{ml}$, streptomycin $100 \mathrm{mg} / \mathrm{ml}$, sodium pyruvate $1 \mathrm{mM}$ and L-glutamine $2 \mathrm{mM}$ (Biological Industries, Kibbutz Beit Haemek, Israel). HeLa cells stably transfected with cytoCGFP were kindly provided by Douglas Green and Sandy Simon. To induce apoptosis, the cells were incubated with STS (Sigma), or exposed to UV irradiation for $5 \mathrm{~min}$. MG132 (20 $\mu \mathrm{M}$, Sigma) for $6 \mathrm{~h}$ was used to inhibit proteasome activity in cells. To inhibit caspase activity, the cells were incubated with the pan-caspase inhibitor $\mathrm{Q}-\mathrm{VD}(3 \mu \mathrm{M})$ for $2 \mathrm{~h}$ before treatment with pro-apoptotic factors.

Constructs. pEF1-AU5 and pEF1-AU5-ARTS constructs contain an AU5 tag that is attached to the N-terminus of ARTS. ${ }^{20}$ ARTSD4aa - mutant ARTS lacking the first 4 aa was generated in our laboratory. The pSC2-6Myc-ARTS was generated using PCR with the following primers: $F$ BamHI- $5^{\prime}$-TCGAGGATCCATC AAGCGTTTCCTGGAGGACACCACGG-3' and the reverse primer: $p E G$ ARTS-R TACCGCTCGAGCTAGTGGCAGCCCTGCCC. The pEBG mammalian expression construct encoding N-terminus GST fusion protein with or without XIAP was a kind gift from Colin Duckett. mCherry-ARTS borrowing the mCherry tag within the ARTS was produced by GenScript (Piscataway, NJ, USA), while mCherry-SMAC was a kindly gift from Sandy Simon.

Transient transfection of cells. jetPEI (Polyplus Transfection Inc., New York, NY, USA) and Transfectol (GeneChoice, Frederick, MD, USA) were used according to the manufacturer's protocol.

Western blot analysis. Western blot analysis was performed as described in Lotan et al. ${ }^{29}$ Visualization was performed using LAS4000 luminescent image analyzer (Fujifilm, Tokyo, Japan). Densitometry analyses of western blot results were done using TotalLab TL100 graphic software (Nonlinear Dynamics Ltd, Durham, NC, USA).

Transmission electron microscopy imaging of ARTS immunogold labeling. The transmission electron microscopy (TEM) imaging of ARTS immunogold labeling was carried out at the Electron Microscopy Unit, Weizmann Institute of Science, Rehovot, Israel. COS-7 cells were prepared for immunogold labeling according to the Tokuyasu method. For single immunogold labeling, the sections were incubated with anti-ARTS antibody (1:50 dilution), followed by anti-mouse coupled with $9 \mathrm{~nm}$ gold antibody, all in blocking medium ( $5 \% \mathrm{FCS}, 0.15 \%$ glycine in PBS). The sections were washed with PBS and $\mathrm{H} 2 \mathrm{O}$, incubated with $0.3 \%$ uranyl acetate, $1.8 \%$ methylcellulose on ice and air dried. Staining using only secondary antibody was used as negative control. The immunogold-labeled sections were examined in an electron microscope (Tecnai-12, FEI, Eindhoven, Netherlands, operating at $120 \mathrm{kV}$ ).

Mitochondria isolation for localization assays. Mitochondriaenriched fraction was obtained by cell homogenization with Dounce homogenizer in $20 \mathrm{mM}$ HEPES-KOH pH 7.5, $10 \mathrm{mM} \mathrm{KCl}, 1.5 \mathrm{mM} \mathrm{MgCl} 2,1 \mathrm{mM}$ sodium EDTA, $1 \mathrm{mM}$ sodium EGTA and $1 \mathrm{mM}$ DTT in the presence of $250 \mathrm{mM}$ sucrose and protease inhibitors (Mini-Complete, Roche Diagnostocs, Indianapolis, IN, USA). Homogenates were centrifuged at $500 \times g$ for $5 \mathrm{~min}$ at $4^{\circ} \mathrm{C}$, and the supernatant was centrifuged at $10000 \times g$ for 20 min to obtain mitochondria. Mitochondria were washed with homogenization buffer.

PK treatment of mitochondrial fractions. Purified mitochondria were resuspended in Tris buffer ( $30 \mathrm{mM}$ Tris $\mathrm{pH} 7.6,1 \mathrm{mM} \mathrm{CaCl}$ ). The suspension was divided into equal aliquots for PK treatment. Aliquots of mitochondria were treated with 50 and $400 \mu \mathrm{g} / \mathrm{ml} \mathrm{PK}$ in a total volume of $100 \mu \mathrm{l}$. PK digestion reactions were incubated in ice for $30 \mathrm{~min}$. PMSF was added to a final concentration of $2 \mathrm{mM}$ to terminate the PK activity, followed by $5 \mathrm{~min}$ of incubation. Digested mitochondria were pelleted by centrifugation at $13000 \times g$ for $20 \mathrm{~min}$, washed in homogenization buffer and resuspended in SDS-PAGE sample buffer. To confirm the activity of the PK, the mitochondrial fraction was solubilized with $1 \%$ Triton X-100, incubated with PK, centrifuged at $13000 \times g$, and the supernatants were analyzed by western blotting as described previously.

Digitonin permeabilization. For digitonin permeabilization, isolated mitochondria were incubated with $0-6 \mathrm{mg} / \mathrm{ml}$ digitonin (final concentration) for $30 \mathrm{~min}$ on ice. Following digitonin treatment, the samples were centrifuged at $16000 \times g$ for $20 \mathrm{~min}$ at $4^{\circ} \mathrm{C}$. The supernatant was subjected to SDS-PAGE and western blotting.

Carbonate extraction of mitochondria. Mitochondria-enriched fraction, which was prepared as described above, was resuspended in homogenization buffer and $10 \mu$ were further divided into half for carbonate treatment. In all, $5 \mu$ of mitochondria suspension was treated with $20 \mu \mathrm{l}$ of $0.1 \mathrm{M}$ sodium carbonate $\mathrm{pH} 11.5$ whereas the other $5 \mu \mathrm{l}$ mitochondrial suspension was mock treated with $20 \mu \mathrm{l}$ sucrose $0.25 \mathrm{M}$ in $0.1 \mathrm{M}$ Tris- $\mathrm{HCl} \mathrm{pH} 7.5$ for $30 \mathrm{~min}$ in ice. Supernatants were retained after treatment by centrifugation at 50000 r.p.m. for $15 \mathrm{~min}$ at $4^{\circ} \mathrm{C}$ and were resuspended in SDS-PAGE sample buffer. The pellets were washed in homogenization buffer and resuspended in SDS-PAGE sample buffer.

Fluorescence assay. For all experiments, cells were seeded in 24-well plates on coverglasses previously coated with fibronectin $(5 \mu \mathrm{g} / \mathrm{ml}$, Biological Industries). Following apoptotic induction $(1.75 \mu \mathrm{M}$ STS), cells were fixed with $4 \%$ paraformaldehyde in PBS for 20 min at room temperature and washed with PBS. For fluorescence assay, HeLa cells were transiently transfected with mCherryARTS or mCherry-SMAC and stained with DAPI (\#157574, MP). Image analysis was carried out using fluorescent microscope (Nikon 50i, Kawasaki, Kanagawa, Japan). For IF assays, HeLa cells were co-transfected with AU5-ARTS and FlagSMAC and stained with MitoTracker Red (M-7512, Molecular Probes, Eugene, OR, USA). HeLa cells stably transfected with cytoC-GFP were used for visualization of endogenous proteins. In both experiments, IF staining were preformed with antiARTS and anti-SMAC antibodies followed by fluorescent secondary antibodies (Jackson ImmunoResearch Laboratories, West Grove, PA, USA). Visualization was carried out using Nikon 50i fluorescent microscope for endogenous proteins, and with Zeiss AxioPhot (Carl Zeiss AG, Feldbach, Switzerland) for the transfected proteins.

GST pull-down binding studies. COS-7 cells were co-transfected with GST empty vector, GST-XIAP or GST-cIAP1 (in pEBG, mammalian GST expression vector) together with pCS2-6Myc-ARTS. The cells were lysed in RIPA buffer $(150 \mathrm{mM} \mathrm{NaCl}, 50 \mathrm{mM}$ Tris-HCl pH 8, 1\% NP-40, 0.5\% deoxycholate acid containing protease inhibitors; Mini-Complete, Roche). The samples were left rotating for $4 \mathrm{~h}$ at $4^{\circ} \mathrm{C}$ with the GST fusion proteins coupled with the glutathione beads. Samples were centrifuged at 4000 r.p.m. at $4^{\circ} \mathrm{C}$ for $4 \mathrm{~min}$ and washed five times in lysis buffer. Proteins were eluted from beads following $5 \mathrm{~min}$ of boiling in sample buffer and separated by SDS-PAGE, followed by western blot analysis.

Cell fractionation by digitonin. Following induction of apoptosis, cytosolic fraction was generated using a digitonin-based subcellular fractionation technique, as described in Adrain et al. ${ }^{38}$ Special care was taken while performing this procedure to avoid translocation of ARTS from mitochondria to the cytosol at time 0 , when cells were not treated with pro-apoptotic inducer, and ARTS was localized to the MOM. Briefly, cells were harvested and centrifuged at $300 \times g$ for $10 \mathrm{~min}$, washed in TBS $2.5 \mathrm{mM} \mathrm{pH} 7.5$ and repelletted. Cells were permeabilized for $5 \mathrm{~min}$ on ice with cytosolic extraction buffer $(250 \mathrm{mM}$ sucrose, $70 \mathrm{mM} \mathrm{KCl}, 137 \mathrm{mM} \mathrm{NaCl}$, 4.3 mM Na2HPO4, $1.4 \mathrm{mM} \mathrm{KH2PO4} \mathrm{pH} \mathrm{7.2,} \mathrm{protease} \mathrm{inhibitor} \mathrm{cocktail;} \mathrm{Complete,}$ Roche) containing freshly prepared digitonin ( $200 \mu \mathrm{g} / \mathrm{ml}, \mathrm{D}-5628$, Sigma). Cytosolic fraction was isolated by collecting the supernatant after centrifugation at $1000 \times g$ 
for $5 \mathrm{~min}$ at $4^{\circ} \mathrm{C}$. Cytosolic fraction was separated by SDS-PAGE and transferred onto nitrocellulose membranes.

Generation of ARTS KD constructs. Four small interference RNA sequences were identified according to the Whitehead siRNA Selection Web Server and Oligoengine shockwave program as follows.

shRNA1: $\quad 5^{\prime}$-gatccccGCCTGAGGCTCCTGGCACCttcaagagaGGTGCCAGGAGC CTCAGGCttttta- $3^{\prime}$ and complementary strand, $5^{\prime}$-agcttaaaaaGCCTGAGGCTCCTGG CACCtctcttgaaGG TGCCAGGAGCCTCAGGCggg- ${ }^{\prime}$

shRNA2: $5^{\prime}$-gatccccGGGAACAGGCCAAGACACTtcaagagaG TGCTCTTGGCC TGTTCCCtttta- $3^{\prime}$ and complementary strand, $5^{\prime}$-agcttaaaaaGGGAACAGGCCAA GAGCACtctcttgaaGTGCTCTTG GCCTGTTCCCggg-3';

shRNA3: $5^{\prime}$-gatccccCAGGCCAAGAGCACCAGGGttcaagagaCCCTGGTGCTC TTGGCCTGtttta- $3^{\prime}$ and complementary strand, $5^{\prime}$-agcttaaaaaCAGGCCAAGAG CACCAGGGtctcttgaaCCCTGGT GCTCTTGGCCTGggg-3';

shRNA4: 5 -gatccccGAGCACCAGGGGCAGGGCTttcaagagaAGCCCTGCCC CTGGTGCTCtttta- $3^{\prime}$ and complementary strand, $5^{\prime}$-agcttaaaaaGAGCACCAGGG GCAGGGCTtctcttgaaAGCCCTGCCC CTGGTGCTCggg-3';

shRNA for lacZ (control shRNA): 5'-gatcccCCTTAATCGCCTTGCAGCACttcaa gagaGTGCTGC AAGGCGATTAAGtttta- $3^{\prime}$ and complementary strand, $5^{\prime}$-agctta aaaaCTTAATCGCCTTGCAGCACtctcttgaaGTGCTGCAAG GCGATTAAGggg-3'.

The $3^{\prime}$-and $5^{\prime}$-ends of the oligonucleotide primers were adapted for cloning into the Bgll and Hindll site of the pSUPER vector. Equimolar amounts of oligonucleotide pairs were annealed and ligated into pSUPER digested with Bgll and Hindll. Positive clones were sequenced to ensure accuracy of RNA interference. The efficiency of RNA interference was initially tested by transfecting each shRNA construct into HeLa cells and performing western blot analysis. shRNA4 reduced ARTS protein levels when compared with control cells.

Generation of ARTS KD HeLa cells. To generate HeLa cells stably expressing ARTS shRNA, subconfluent cultures were transfected with shRNA4 construct or the control shRNA construct ( $2 \mu \mathrm{g}$ per $35 \mathrm{~mm}$ plate), together with pCDNA 3.1 for selection (1:20 ratio). Transfection was performed with jetPEl reagent (Polyplus transfection) according to the manufacturer's instructions. Following selection with $0.5 \mathrm{mg} / \mathrm{ml} \mathrm{G} 418$ (Sigma), mass cultures were tested for the reduction of endogenous ARTS protein. These cells were used in this study.

BiFC assay examining ARTS-XIAP direct binding. The split-Venus, BiFC system ${ }^{31}$ was used to evaluate the kinetics of ARTS and XIAP complex formation in HeLa cells following STS treatment. For this purpose, ARTS and XIAP were fused to the split-Venus proteins (a brighter and more photostable variant of YFP) - VC 155-239 and VN 1-173, respectively. These constructs were expressed by transient transfection in HeLa cells together with pdsRED plasmid, which was used as a transfection efficiency marker.

Flow cytometry analysis of BiFC-transfected cells. Transfection was performed using $200 \mathrm{ng}$ of each plasmid along with $50 \mathrm{ng}$ of pdsRED. Thirty-six hours later, $1.75 \mu \mathrm{M}$ STS was added to HeLa cells for the indicated time periods to induce apoptosis. Cells were trypsinized, washed with PBS and resuspended in $0.5 \mathrm{ml}$ PBS. Flow cytometry analyses of cell suspensions were performed using FACS (FACSCantoll; BD Biosciences, San Jose, CA, USA) equipped with an argon laser emitting at $488 \mathrm{~nm}$. Analysis was restricted to live cells. Results were analyzed using FACSDIVA software (BD Biosciences). To normalize the results according to transfection efficiency, ratio between YFP and Red fluorescence was calculated for each time point and represented relatively to that without apoptotic induction $(t=0)$.

IF analysis of BiFC-transfected cells. HeLa cells were seeded on coverslides placed in 24-well plates. Cells were transfected with $100 \mathrm{ng}$ of each plasmid (XIAP-VN and ARTS-VC) along with $25 \mathrm{ng}$ of pdsRED. Thirty-six hours after transfection, the cells were treated with STS for the indicated time periods to induce apoptosis. Then, cells were fixed in 4\% PFA and washed with PBS. Image analysis was carried out using confocal laser microscopy (Zeiss LSM 510, Carl Zeiss $A G)$.

Caspase-9 activity assay. Caspase- 9 activity was determined in HeLa and ARTS KD HeLa cells using measuring cleavage of fluorescently labeled substrate of caspase-9 (Caspase-9 Fluorometric Assay Kit, K118, BioVision, Mountain View, CA, USA) according to the manufacturer's instructions.
In situ cell death detection assay - TUNEL. HeLa and ARTS KD HeLa cells were treated with STS for different time periods. TUNEL assay (In Situ Cell Death Detection Kit; Roche, \#12-156-792-910) was performed on the slides followed by image analysis using fluorescent microscopy (Zeiss AxioPhot). DAPI stain (MP, 157574) was used to assess total cell number. Ratio of TUNEL-positive cells out of total cells represented the number of apoptotic cells. The percentage of TUNEL-positive cells was calculated as the number of TUNEL-positive cells divided by the total number of cells.

XTT viability assay. XTT cell viability assay was performed in 96-well plate according to the manufacturer's instructions (Cell proliferation kit - XTT-based colourometric assay, Biological Industries), following STS $(1.75 \mu \mathrm{M})$ treatment of cells for the indicated time periods. Absorbance of the water-soluble formazan salt was measured on PowerWaveXS Elisa reader (BioTek, Winooski, VT, USA) at primary wavelength of $450 \mathrm{~nm}$ and a reference wavelength of $630 \mathrm{~nm}$. Each plate contained the samples, positive control, negative control and blank. The assay was performed in quadricates and the results were presented as a ratio values between each sample and the negative control \pm S.E.

Clonogenic assays. Apoptosis was induced in HeLa and ARTS KD HeLa cells by $1.75 \mu \mathrm{M}$ STS. Then, cells were split and 300 cells were seeded in $6-\mathrm{cm}$ dishes and allowed to grow for 12 days. Colonies $>50$ cells were counted.

Survival assay. HeLa and ARTS KD HeLa cells were treated with STS for different time periods. In all, $4 \%$ paraformaldehyde was used to fix the cells for $30 \mathrm{~min}$, followed by two repeated washes with PBSx1. Permeabilization was performed using $0.5 \%$ Triton X-100 for 5 min. Staining with DAPI (MP, 157574) and scoring cells were done using fluorescent microscope Nikon 50i. Counts for each sample represent the average number of viable cells \pm S.E. in eight different fields.

\section{Conflict of Interest}

The authors declare no conflict of interest.

Acknowledgements. We thank John Silke, Colin Duckett, Sandy Simon, Chunying Du, Douglas Green and Changdeng Hu for generously providing us with materials. We are very grateful to Michael Loewenstein, Sagie Schif-Zuck, Alana Bahjan Persaud and Travis Gorenc for technical assistance and to Hermann Steller for critical reading of this manuscript. This work was supported by funds from BSF (US Israel Binational Science Foundation) grant \#2003085 (to SL), ISF (Israel Science Foundation) grant \#1264/06 (to SL), a grant from Israel Cancer Association (ICA) (to SL), and by a generous donation from the Charles Wolfson Charitable Trust, England.

1. Shi Y. Mechanisms of caspase activation and inhibition during apoptosis. Mol Cell 2002; 9 : 459-470.

2. Green DR, Kroemer G. The pathophysiology of mitochondrial cell death. Science 2004; 305: 626-629.

3. Rodriguez J, Lazebnik Y. Caspase-9 and APAF-1 form an active holoenzyme. Genes Dev 1999; 13: 3179-3184.

4. Salvesen GS, Duckett CS. IAP proteins: blocking the road to death's door. Nat Rev Mol Cell Biol 2002; 3: 401-410.

5. Gyrd-Hansen M, Darding M, Miasari M, Santoro MM, Zender L, Xue W et al. IAPs contain an evolutionarily conserved ubiquitin-binding domain that regulates NF-kappaB as well as cell survival and oncogenesis. Nat Cell Biol 2008; 10: 1309-1317.

6. Rajalingam K, Dikic I. Inhibitors of apoptosis catch ubiquitin. Biochem J 2009; 417: e1-e3.

7. Schile AJ, Garcia-Fernandez M, Steller H. Regulation of apoptosis by XIAP ubiquitin-ligase activity. Genes Dev 2008; 22: 2256-2266.

8. Deveraux QL, Takahashi R, Salvesen GS, Reed JC. X-linked IAP is a direct inhibitor of celldeath proteases. Nature 1997; 388: 300-304.

9. Wright CW, Duckett CS. Reawakening the cellular death program in neoplasia through the therapeutic blockade of IAP function. J Clin Invest 2005; 115: 2673-2678.

10. Ryoo HD, Bergmann A, Gonen H, Ciechanover A, Steller H. Regulation of Drosophila IAP1 degradation and apoptosis by reaper and ubcD1. Nat Cell Biol 2002; 4: 432-438.

11. Kornbluth $\mathrm{S}$, White K. Apoptosis in Drosophila: neither fish nor fowl (nor man, nor worm). J Cell Sci 2005; 118 (Pt 9): 1779-1787.

12. Potts PR, Singh S, Knezek M, Thompson CB, Deshmukh M. Critical function of endogenous XIAP in regulating caspase activation during sympathetic neuronal apoptosis. J Cell Biol 2003; 163: 789-799. 
13. Jost PJ, Grabow $S$, Gray D, McKenzie MD, Nachbur U, Huang DC et al. XIAP discriminates between type I and type II FAS-induced apoptosis. Nature 2009; 460: 1035-1039.

14. Du C, Fang M, Li Y, Li L, Wang X. Smac, a mitochondrial protein that promotes cytochrome c-dependent caspase activation by eliminating IAP inhibition. Cell 2000; 102: 33-42.

15. Verhagen AM, Ekert PG, Pakusch M, Silke J, Connolly LM, Reid GE et al. Identification of DIABLO, a mammalian protein that promotes apoptosis by binding to and antagonizing IAP proteins. Cell 2000; 102: 43-53.

16. van Loo G, van Gurp M, Depuydt B, Srinivasula SM, Rodriguez I, Alnemri ES et al. The serine protease $\mathrm{Omi} / \mathrm{HtrA} 2$ is released from mitochondria during apoptosis. Omi interacts with caspase-inhibitor XIAP and induces enhanced caspase activity. Cell Death Differ 2002; 9: 20-26.

17. Gottfried $\mathrm{Y}$, Rotem A, Lotan $\mathrm{R}$, Steller $\mathrm{H}$, Larisch $\mathrm{S}$. The mitochondrial ARTS protein promotes apoptosis through targeting XIAP. EMBO J 2004; 23: 1627-1635.

18. Larisch S, Yi Y, Lotan R, Kerner H, Eimerl S, Tony Parks $W$ et al. A novel mitochondrial septin-like protein, ARTS, mediates apoptosis dependent on its P-loop motif. Nat Cell Biol 2000; 2: 915-921.

19. Okada H, Suh WK, Jin J, Woo M, Du C, Elia A et al. Generation and characterization of Smac/DIABLO-deficient mice. Mol Cell Biol 2002; 22: 3509-3517.

20. Martins LM, Morrison A, Klupsch K, Fedele V, Moisoi N, Teismann P et al. Neuroprotective role of the Reaper-related serine protease HtrA2/Omi revealed by targeted deletion in mice. Mol Cell Biol 2004; 24: 9848-9862.

21. Elhasid R, Sahar D, Merling A, Zivony Y, Rotem A, Ben-Arush M et al. Mitochondrial proapoptotic ARTS protein is lost in the majority of acute lymphoblastic leukemia patients. Oncogene 2004; 23: 5468-5475.

22. Garcia-Fernandez M, Kissel H, Brown S, Gorenc T, Schile AJ, Rafii S et al. Sept4/ARTS is required for stem cell apoptosis and tumor suppression. Genes Dev 2010; 24: 2282-2293.

23. Kissel H, Georgescu MM, Larisch S, Manova K, Hunnicutt GR, Steller H. The Sept4 septin locus is required for sperm terminal differentiation in mice. Dev Cell 2005; 8: 353-364.

24. Tait SW, Green DR. Mitochondria and cell death: outer membrane permeabilization and beyond. Nat Rev Mol Cell Biol 2010; 11: 621-632.

25. Burri L, Strahm Y, Hawkins CJ, Gentle IE, Puryer MA, Verhagen A et al. Mature DIABLO/ Smac is produced by the IMP protease complex on the mitochondrial inner membrane. Mol Biol Cell 2005; 16: 2926-2933.

26. Garrido C, Galluzzi L, Brunet M, Puig PE, Didelot C, Kroemer G. Mechanisms of cytochrome c release from mitochondria. Cell Death Differ 2006; 13: 1423-1433.
27. Lotan R, Rotem A, Gonen H, Finberg JP, Kemeny S, Steller $\mathrm{H}$ et al. Regulation of the proapoptotic ARTS protein by ubiquitin-mediated degradation. J Biol Chem 2005; 280 25802-25810.

28. Munoz-Pinedo C, Guio-Carrion A, Goldstein JC, Fitzgerald P, Newmeyer DD, Green DR. Different mitochondrial intermembrane space proteins are released during apoptosis in a manner that is coordinately initiated but can vary in duration. Proc Natl Acad Sci USA 2006; 103: $11573-11578$

29. Shyu YJ, Hu CD. Fluorescence complementation: an emerging tool for biological research Trends Biotechnol 2008; 26: 622-630.

30. Gross A, Yin XM, Wang K, Wei MC, Jockel J, Milliman C et al. Caspase cleaved BID targets mitochondria and is required for cytochrome $c$ release, while $B C L-X L$ prevents this release but not tumor necrosis factor-R1/Fas death. J Biol Chem 1999; 274: 1156-1163.

31. Li H, Zhu H, Xu CJ, Yuan J. Cleavage of BID by caspase 8 mediates the mitochondrial damage in the Fas pathway of apoptosis. Cell 1998; 94: 491-501.

32. Hayakawa A, Kawamoto Y, Nakajima H, Sakai J, Takasawa R, Nakashima I et al. Bid truncation mediated by caspases-3 and -9 in vinorelbine-induced apoptosis. Apoptosis 2008; 13: 523-530.

33. Yang QH, Du C. Smac/DIABLO selectively reduces the levels of C-IAP1 and C-IAP2 but not that of XIAP and livin in HeLa cells. J Biol Chem 2004; 279: 16963-16970.

34. Rehm M, Huber HJ, Dussmann $\mathrm{H}$, Prehn JH. Systems analysis of effector caspase activation and its control by X-linked inhibitor of apoptosis protein. EMBO J 2006; 25: 4338-4349.

35. Adrain C, Creagh EM, Martin SJ. Apoptosis-associated release of Smac/DIABLO from mitochondria requires active caspases and is blocked by Bcl-2. EMBO J 2001; 20 $6627-6636$.

36. Braun T, Dar S, Vorobiov D, Lindenboim L, Dascal N, Stein R. Expression of Bcl-x(S) in Xenopus oocytes induces BH3-dependent and caspase-dependent cytochrome $\mathrm{c}$ release and apoptosis. Mol Cancer Res 2003; 1: 186-194.

37. Bhola PD, Mattheyses AL, Simon SM. Spatial and temporal dynamics of mitochondria membrane permeability waves during apoptosis. Biophys J 2009; 97: 2222-2231.

38. Garrison JB, Correa RG, Gerlic M, Yip KW, Krieg A, Tamble CM et al. ARTS and Siah collaborate in a pathway for XIAP degradation. Mol Cell 2011; 41: 107-116.

39. Martinou JC, Youle RJ. Which came first, the cytochrome $c$ release or the mitochondrial fission? Cell Death Differ 2006; 13: 1291-1295.

40. Bornstein B, Gottfried Y, Edison N, Shekhtman A, Lev T, Glaser F et al. ARTS binds XIAP by a distinct mechanism. Apoptosis 2011; 16: 869-881.

\section{Supplementary Information accompanies the paper on Cell Death and Differentiation website (http://www.nature.com/cdd)}

